\title{
Neutrino Mass, Coupling Unification, Verifiable Proton Decay, Vacuum Stability, and WIMP Dark Matter in SU(5)
}

\author{
Biswonath Sahoo, Mainak Chakraborty, and M. K. Parida (i) \\ Centre of Excellence in Theoretical and Mathematical Sciences, Siksha 'O'Anusandhan (Deemed to be University), \\ Khandagiri Square, Bhubaneswar 751030, Odisha, India
}

Correspondence should be addressed to M. K. Parida; minaparida@soa.ac.in

Received 5 April 2018; Accepted 24 May 2018; Published 6 August 2018

Academic Editor: Farinaldo Queiroz

Copyright (c) 2018 Biswonath Sahoo et al. This is an open access article distributed under the Creative Commons Attribution License, which permits unrestricted use, distribution, and reproduction in any medium, provided the original work is properly cited. The publication of this article was funded by $\mathrm{SCOAP}^{3}$.

\begin{abstract}
Nonsupersymmetric minimal SU(5) with Higgs representations $24_{H}$ and $5_{H}$ and standard fermions in $\overline{5}_{F} \oplus 10_{F}$ is well known for its failure in unification of gauge couplings and lack of predicting neutrino masses. Like standard model, it is also affected by the instability of the Higgs scalar potential. We note that extending the Higgs sector by $75_{H}$ and $15_{H}$ not only leads to the popular type-II seesaw ansatz for neutrino masses with a lower bound on the triplet mass $M_{\Delta}>2 \times 10^{9} \mathrm{GeV}$, but also achieves precision unification of gauge couplings without proliferation of nonstandard light Higgs scalars or fermions near the TeV scale. Consistent with recent LUX-2016 lower bound, the model easily accommodates a singlet scalar WIMP dark matter near the TeV scale which resolves the vacuum stability issue even after inclusion of heavy triplet threshold effect. We estimate proton lifetime predictions for $p \longrightarrow e^{+} \pi^{0}$ including uncertainties due to input parameters and threshold effects due to superheavy Higgs scalars and superheavy $X^{ \pm 4 / 3}, Y^{ \pm 1 / 3}$ gauge bosons. The predicted lifetime is noted to be verifiable at Super Kamiokande and Hyper Kamiokande experiments.
\end{abstract}

\section{Introduction}

Standard model (SM) of strong and electroweak interactions has been established by numerous experimental tests, yet evidences on neutrino mass [1-5], the phenomena of dark matter [6-25] , and baryon asymmetry of the universe (BAU) $[8,26-29]$ call for beyond standard model (BSM) physics. It is well known that grand unified theories (GUTs) [30-37] are capable of addressing a number of limitations of the SM effectively. There are interesting theories on neutrino mass generation mechanisms [38-45] based upon various seesaw mechanisms such as type-I, type-II, type-III [46-62], linear $[63,64]$, and inverse [65-75]. Interesting models for Dirac neutrino mass origin of the neutrino oscillation data have been also proposed $[76,77]$. In the absence of experimental evidence of supersymmetry so far, nonsupersymmetric (nonSUSY) GUTs are being extensively exploited by reconciling to the underlying gauge hierarchy problem through finetuning $[78,79]$. Higher rank GUTs like $S O(10)$ and $E_{6}$ can not define a unique symmetry breaking path to the SM gauge theory because of large number of possibilities with one and more intermediate symmetry breakings consistent with electroweak precision data on $\sin ^{2} \theta_{W}\left(M_{Z}\right), \alpha_{S}\left(M_{Z}\right)$, and $\alpha\left(M_{Z}\right)$ [80-82]. On the other hand, the rank-4 minimal SU(5) [32] with Higgs representations $5_{H}$ and $24_{H}$ defines only one unique symmetry breaking path to the standard model

$$
S U(5) \longrightarrow S M
$$

Type-I seesaw [46-52] needs nonstandard heavy righthanded neutrino, linear and inverse seesaw both need nonstandard fermions and scalars, and type-III seesaw [38-45, 58-62] needs only nonstandard fermionic extension for their implementation. Out of these popular seesaw mechanisms, type-II seesaw mechanism is the one which needs a heavy nonstandard triplet scalar [52-57, 59]. With a second triplet scalar, it is also capable of predicting baryon asymmetry of the universe [57] which is one of the main motivations behind this investigation. This neutrino mass generation mechanism, gauge coupling unification, dark matter, and vacuum stability are the focus of the present work. 
Like the minimal SM, with its 15 fermions per generation and the standard Higgs doublet $\phi(2,1 / 2,1)$, the minimal SU(5) with Higgs representations $5_{H}$ and $24_{H}$ predicts neutrinos to be massless subject to a tiny $\mathcal{O}\left(10^{-5}\right) \mathrm{eV}$ contribution due to nonrenormalizable Planck scale effect which is nearly 4 orders smaller than the requirement of neutrino oscillation data. As the particle spectrum below the GUT symmetry breaking scale is identically equal to the SM spectrum, like SM, the minimal GUT fails to unify gauge couplings [8387]. Also it predicts instability of the Higgs quartic coupling at mass scales $\mu \geq 5 \times 10^{9} \mathrm{GeV}$ [88-90] after which the coupling continues to be increasingly negative at least up to the unification scale.

A number of interesting models have been suggested for coupling unification by populating the grand desert and for enhancing proton lifetime predictions [60-62, 91-98]. In these models a number of fermion or scalar masses below the GUT scale have been utilised to achieve unification. Interesting possibility of type-III seesaw [60-62] with experimentally verifiable dilepton production [99] at LHC has been also investigated.

The other shortcoming of minimal non-SUSY SU(5) is its inability to predict dark matter which appears to belong to two distinct categories: (i) the weakly interacting massive particle (WIMP) dark matter of bounded mass $<100 \mathrm{TeV}$ and (ii) the decaying dark matter which has been suggested to be a possible source of $\mathrm{PeV}$ energy IceCube neutrinos.

In this work we implement a novel mechanism for coupling unification and neutrino masses together. When SU(5) is extended by the addition of its Higgs representations $75_{H}$ and $15_{H}$, it achieves two objectives: (i) neutrino mass and mixing generation through type-II seesaw mechanism and (ii) precision gauge coupling unification with experimentally accessible proton lifetime.

But this does not cure the vacuum instability problem persisting in the model as well as the need for WIMP dark matter prediction. Out of these two, as we note in this work, when the dark matter prediction is successfully inducted into the model, the other problem on vacuum stability is automatically resolved.

In contrast to the popular belief on low proton lifetime prediction of the minimal SU(5) [35], we estimate new precise and enhanced predictions of this model including threshold effects [100-108] of heavy particles near the GUT scale. Predicted lifetimes are found to be within the accessible ranges of Superkamiokande and Hyperkamiokande experimental search programmes [109].

This paper is organised in the following manner. In Section 2 we discuss neutrino mass generation mechanism in extended SU(5). Section 3 deals with the problem of gauge coupling unification. In Section 4 we make proton lifetime prediction including possible uncertainties. Embedding WIMP scalar DM in SU(5) is discussed in Section 5 with a brief outline on the current experimental status. Resolution of vacuum stability issue is explained in Section 6. We summarise and conclude in Section 7. Renormalization group equations for gauge and Higgs quartic couplings are discussed in the Appendix.

\section{Neutrino Mass Through Type-II Seesaw in SU(5)}

As noted in Section 1, in contrast to many possible alternative symmetry breaking paths to SM from non-SUSY SO(10) and $E_{6}$ [80-82], SU(5) predicts only one symmetry breaking path which enhances its verifiable predictive capability. Fifteen SM fermions are placed in two different $S U(5)$ representations:

$$
\begin{aligned}
\overline{5}_{F} & =\left(\begin{array}{c}
d_{1}^{C} \\
d_{2}^{C} \\
d_{3}^{C} \\
e^{-} \\
-v_{e}
\end{array}\right){ }_{L} \\
10_{F} & =\left(\begin{array}{ccccc}
0 & u_{2}^{C} & -u_{3}^{C} & u_{1} & d_{1} \\
-u_{2}^{C} & 0 & u_{1}^{C} & u_{2} & d_{2} \\
u_{3}^{C} & -u_{1}^{C} & 0 & u_{3} & d_{3} \\
-u_{1} & -u_{2} & -u_{3} & 0 & e^{C} \\
-d_{1} & -d_{2} & -d_{3} & -e^{C} & 0
\end{array}\right) .
\end{aligned}
$$

Lack of RH $v$ in these representations gives vanishing Dirac neutrino mass and vanishing Majorana neutrino mass at renormalizable level. Planck scale induced small Majorana masses can be generated through nonrenormalizable dim. 5 interaction

$$
-\mathscr{L}_{\text {NR }}=\frac{\kappa_{i j}}{M_{\text {Planck }}} \overline{5}_{F_{i}} \overline{5}_{F_{j}} 5_{H} 5_{H}+\text { h.c, }
$$

leading to $m_{v} \sim 10^{-5} \mathrm{eV}$ which is too low to explain neutrino oscillation data. Mechanism of Dirac neutrino mass generation has been discussed $[76,77]$ matching the neutrino oscillation data. Using extensions of the minimal GUT typeIII seesaw origin of neutrino mass has been discussed where the nonstandard fermionic triplet $\Sigma_{F}(3,0,1)$ mediates the seesaw. This model can be experimentally tested by the production of like-sign dilepton signals at LHC.

Type-II seesaw mechanism for neutrino mass [53-57] does not need any nonstandard fermion but needs only the nonstandard left-handed Higgs scalar triplet $\Delta_{L}(3,-1,1)$ with $Y=-2$ which directly couples with the a dilepton pair. It also directly couples to standard Higgs doublet $\phi$. As such the standard Higgs VEV can be transmitted as a small induced VEV generating Majorana mass term for the light neutrinos. As this $\Delta_{L}(3,-1,1)$ is contained in the symmetric $\mathrm{SU}(5)$ scalar representation $15_{H}$, the scalar sector of the minimal GUT needs to include $15_{H}$ in addition to $5_{H}$ and $24_{H}$.

The Yukawa Lagrangian

$$
-\mathscr{L}^{(I I)}=l_{L_{i}}^{T} \operatorname{Ci\tau } \tau_{2} Y_{i j}\left(\frac{\vec{\tau} \cdot \overrightarrow{\Delta_{L}}}{\sqrt{2}}\right)^{\dagger} l_{L_{j}}+h . c,
$$

combined with the relevant part of the Higgs potential

$$
\mathscr{V}_{I I}=M_{\Delta}^{2} \operatorname{Tr}\left[\Delta_{L}^{\dagger} \Delta_{L}\right]+\mu_{\Delta} \tilde{\phi}^{\dagger}\left(\frac{\vec{\tau} \cdot \overrightarrow{\Delta_{L}}}{\sqrt{2}}\right) \phi+\text { h.c. }
$$


gives rise to the type-II seesaw contribution. In our notation $l_{L_{i}}^{T}=\left(\nu_{L_{i}}, e_{L_{i}}\right)(i=$ generation index $)$ and $\phi^{T}=\left(\phi^{+}, \phi^{0}\right)$ which are the lepton and scalar doublet of $S U(2)_{L}$. Here $\widetilde{\phi}=i \tau_{2} \phi^{*}$, $\vec{\tau}=\left(\tau_{1}, \tau_{2}, \tau_{3}\right)\left(\tau_{i}\right.$ are the $2 \times 2$ Pauli spin matrices $)$ and, similarly, the scalar triplet $\Delta_{L}$ in the adjoint representation of $S U(2)_{L}$ is expressed as $\overrightarrow{\Delta_{L}}=\left(\Delta_{L}^{1}, \Delta_{L}^{2}, \Delta_{L}^{3}\right)$. The Majorana type Yukawa coupling $Y$ is a $3 \times 3$ matrix in flavor space and $C$ is the charge conjugation matrix. Then

$$
\begin{aligned}
\left(\frac{\vec{\tau} \cdot \overrightarrow{\Delta_{L}}}{\sqrt{2}}\right) & =\frac{1}{\sqrt{2}}\left(\tau_{1} \Delta_{L}^{1}+\tau_{2} \Delta_{L}^{2}+\tau_{3} \Delta_{L}^{3}\right) \\
& =\left(\begin{array}{cc}
\frac{\Delta^{+}}{\sqrt{2}} & \Delta^{++} \\
\Delta^{0} & -\frac{\Delta^{+}}{\sqrt{2}}
\end{array}\right)_{L}
\end{aligned}
$$

where different components are given by

$$
\begin{aligned}
\Delta_{L}^{0} & =\frac{1}{\sqrt{2}}\left(\Delta_{L}^{1}+i \Delta_{L}^{2}\right), \\
\Delta_{L}^{+} & =\Delta_{L}^{3}, \\
\Delta_{L}^{++} & =\frac{1}{\sqrt{2}}\left(\Delta_{L}^{1}-i \Delta_{L}^{2}\right)
\end{aligned}
$$

A diagrammatic representation for type-II seesaw generation of neutrino mass is shown in Figure 1. From the Feynman diagram shown in Figure 1 the induced VEV of the scalar triplet is

$$
v_{L}=\frac{\mu_{\Delta} v^{2}}{2 M_{\Delta}^{2}},
$$

leading to the type-II seesaw formula

$$
m_{v}=2 Y v_{L}
$$

It is necessary to explain the origin of the $B-L$ breaking scale $\mu_{\Delta}$ that occurs in (4), (5), and (8) as well as the Feynman diagram of Figure 1. SU(5) invariance permits the triplet coupling $\mu_{\Delta} 15_{H}^{\dagger} 5_{H} 5_{H}$ leading to SM invariant coupling $\mu_{\Delta} \Delta_{L} \phi \phi$. Therefore, in one approach, $\mu_{\Delta}$ may be treated as explicitly lepton number violating parameter. Alternatively, it is also possible to attribute a spontaneous lepton number violating origin to this parameter. Since the SM model gauge theory has to remain unbroken down to the electroweak scale, the lepton number violating scale can be generated by the VEV of a Higgs scalar that transforms as a singlet under SM. Such a singlet $S_{B L}(1,0,1)$ carrying $B-L=-2$ occurs in the Higgs representation $50_{H}[36,110]$. The part of the $S U(5)$ invariant potential that generates this scale is

$$
V_{B L}=\lambda_{5} 50_{H} 15_{H} 5_{H} 5_{H}+\text { h.c, }
$$

leading to $\mu_{\Delta}=\lambda_{5}<S_{B L}>$. The $U(1)_{B-L}$ symmetric origin of $\mu_{\Delta_{L}}$ becomes more transparent if one treats $\mathrm{SU}(5)$ as the remnant of $S U(5) \times U(1)_{B-L}$ or higher rank GUTs like $S O(10)$ and $E_{6}$. If unification constraint as discussed below is ignored, the order of magnitude of $\mu_{\Delta}$ can be anywhere in the range $\mathcal{O}\left(\mathscr{M}_{\mathrm{W}}\right)-\mathcal{O}\left(\mathscr{M}_{\text {Planck }}\right)$. But as we will find in the subsequent sections, gauge coupling unification in the present $\mathrm{SU}(5)$ framework imposes the lower bound $\mu_{\Delta} \simeq M_{\Delta} \geq 10^{9.23} \mathrm{GeV}$.

\subsection{Type-II Seesaw Fit to the Neutrino Oscillation Data}

2.1.1. Neutrino Mass Matrix from Oscillation Data. The effective light neutrino mass matrix $\left(m_{\gamma}\right)$ is diagonalised by a unitary matrix $U$ (in PMNS parametrisation which is written as $\left.U_{\text {PMNS }}\right)$ and yields three mass eigenvalues $\left(m_{1}, m_{2}, m_{3}\right)$. The light neutrino mass matrix $\left(m_{\nu}\right)$ can be reconstructed as

$$
m_{v}=U_{P M N S} \operatorname{diag}\left(m_{1}, m_{2}, m_{3}\right) U_{P M N S}^{T}
$$

where PMNS matrix is parameterised using the PDG convention [111] as

$$
U_{\mathrm{PMNS}}=\left(\begin{array}{ccc}
c_{12} c_{13} & s_{12} c_{13} & s_{13} e^{-i \delta} \\
-s_{12} c_{23}-c_{12} s_{23} s_{13} e^{i \delta} & c_{12} c_{23}-s_{12} s_{23} s_{13} e^{i \delta} & s_{23} c_{13} \\
s_{12} s_{23}-c_{12} c_{23} s_{13} e^{i \delta} & -c_{12} s_{23}-s_{12} c_{23} s_{13} e^{i \delta} & c_{23} c_{13}
\end{array}\right) \operatorname{diag}\left(e^{i \alpha_{M} / 2}, e^{i \beta_{M} / 2}, 1\right)
$$

where $s_{i j}=\sin \theta_{i j}, c_{i j}=\cos \theta_{i j}$ with $(i, j=1,2,3), \delta$ is the Dirac CP phase, and $\left(\alpha_{M}, \beta_{M}\right)$ are Majorana phases.

Here we present our numerical analysis within $3 \sigma$ and $1 \sigma$ limits of experimental data. As we do not have any experimental information about Majorana phases, they are varied in the whole $2 \pi$ interval randomly. From the set of randomly generated values we pick only one set of $\left(\alpha_{M}, \beta_{M}\right)$ and use them for our numerical estimations. The procedure adopted here can be repeated to derive corresponding solutions for the Majorana coupling matrix $Y$ for other sets of randomly chosen Majorana phases. Although very recently $3 \sigma$ and $1 \sigma$ limits of Dirac CP phase have been announced [1], we prefer to use only their central value as an example. For our present analysis we choose a single set of $\left(\alpha_{M}, \beta_{M}\right)$ from a number of sets derived by random sampling and also a single value of $\delta$ close to the best fit value. For our analysis all possible 


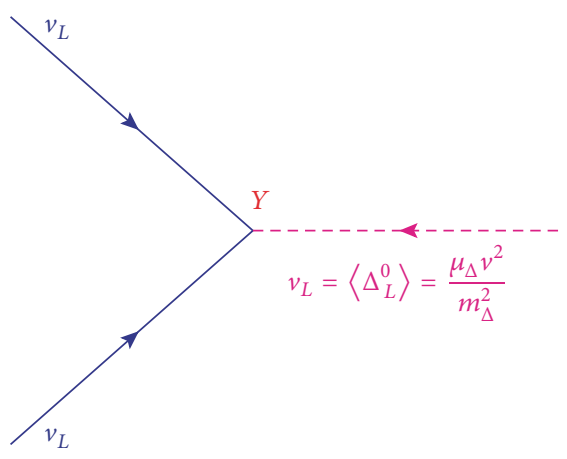

(a)

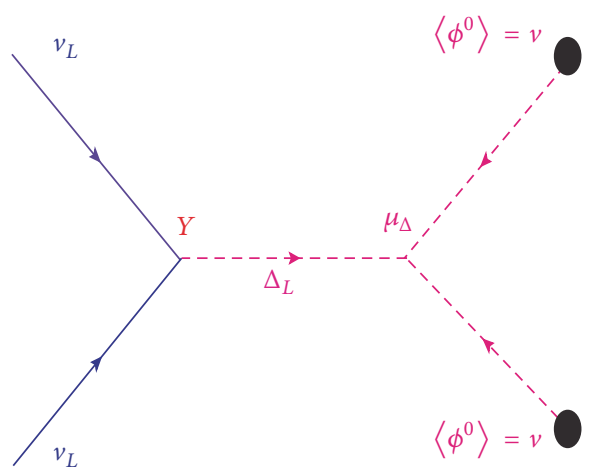

(b)

FIGURE 1: Schematic representation of generation of type-II term corresponding to (4) (a) and combination of (5)(b) where dashed line as triplet propagator supplies the damping factor $M_{\Delta}^{-2}$ to the induced vev $v_{L}$.

values of the solar and atmospheric mass squared differences and mixing angles have been taken which lie within the $3 \sigma$ (or $1 \sigma$ ) limit of the oscillation data as determined by recent global analysis [1]. Summary of the global analysis is presented in Table 1. At first we analyze the limits imposed on the neutrino Yukawa couplings by $3 \sigma$ oscillation constraints taking into account both the mass ordering of light neutrinos, normal ordering (NO), and inverted ordering (IO). In this case we use only one fixed value of the lightest neutrino mass eigenvalue and the other two mass eigenvalues are calculated using the experimental values of the mass squared differences. In this $3 \sigma$ case we represent the bounds on the elements of Yukawa matrix in a tabular form. Later we proceed to estimate the bounds on the $Y$ matrix elements imposed by $1 \sigma$ experimental constraints of oscillation observables. In this analysis instead of fixed lightest neutrino mass eigenvalue, we vary it in the range $(0-0.2) \mathrm{eV}$. The other two mass eigenvalues are calculated using $1 \sigma$ ranges of solar and atmospheric mass squared differences. As already explained we use a single set of randomly chosen $\left(\alpha_{M}, \beta_{M}\right)$ and the central value of $\delta$ quoted in the Table 1 . The variation of $Y$ matrix elements is expressed in terms of their moduli $\left(\left|Y_{i j}\right|\right)$ and the corresponding phases $\left.\left(\phi_{i j}\right)\right)$ with $m_{1}$ are shown graphically in Figures 2, 3, and 4 in the NO case. It is clear from the plots that for each single value of $m_{1}$ there is a band of allowed values of $\left|Y_{i j}\right|$ and $\phi_{i j}$. This band signifies the $1 \sigma$ allowed range of the corresponding matrix element for that single value of $m_{1}$. To represent the $1 \sigma$ bounds in a more transparent manner we produce another set of plots as in Figures 5 and 6 where we show the allowed values of $\left|Y_{i j}\right|$ and $\phi_{i j}$ for a fixed value of $m_{1}$. It is to be noted that in this present work graphical representation is done for normally ordered light neutrinos only. Similar kind of exercise can be carried out for inverted mass ordering also.

\subsubsection{Majorana Yukawa Coupling for $3 \sigma$ Bounds of Neutrino} Oscillation Data. We now estimate the $m_{v}$ matrix for the normally ordered (NO) case. For this purpose we take the mass of the lightest neutrino as $m_{1}=0.00127 \mathrm{eV}$. Then using the $3 \sigma$ ranges of solar and atmospheric mass squared differences for NO case, as mentioned in Table 1, the other two neutrino mass eigenvalues are calculated. Obviously we get a range of values of $m_{2}$ and $m_{3}$. Plugging in these mass eigenvalues along with all possible combinations and the mixing angles within the $3 \sigma$ bound in (11) we obtain large number of sets of $m_{v}$ matrix. Thus we also get respective bounds on the elements of the $m_{v}$ matrix (or equivalently on the Yukawa coupling matrix $Y$ ) corresponding to the $3 \sigma$ oscillation constraints. As mentioned earlier we use single set of randomly chosen Majorana phases while the Dirac $\mathrm{CP}$ phase is chosen close to its central value. The effective light neutrino mass matrix $m_{v}$ and the coupling matrix $Y$ are connected through the induced VEV $v_{l}$ which is obtained by assuming the dimensionful coupling $\mu_{\Delta} \sim M_{\Delta}$ where $M_{\Delta}=$ $10^{12} \mathrm{GeV}$ and the electroweak VEV is $246 \mathrm{GeV}$. With these considerations we estimate the $3 \sigma$ bound on the elements of $Y$ matrix and present them in Table 2. In the inverted mass ordering the smallest mass eigenvalue is $m_{3}$ which is set to be equal to $0.00127 \mathrm{eV}$. The other two eigenvalues are calculated using the $3 \sigma$ limit of the solar and atmospheric mass squared differences. In this case also we are able to put a bound on the modulus and phase of the Yukawa coupling matrix following the same procedure as done in the case of NO. The constrained parameters $\left(\left|Y_{i j}\right|, \phi_{i j}\right)$ for inverted mass ordering are given in Table 3.

As we have taken a most general complex symmetric structure of the $m_{v}$ matrix (or in other words the Yukawa coupling matrix $Y$ ) without imposing any kinds specific flavor symmetry, it does not have any definite prediction of the Dirac CP violating phase $\delta$. Any value of $\delta$ in the given $3 \sigma$ range can be accommodated. In this regard few remarks about the present experimental status of the Dirac CP phase are in order. The recent global analysis of oscillation data done in [1] has made it clear that value of the Dirac CP phase $\delta=\pi / 2$ is more or less ruled out. In normal mass ordering (NO) $\delta=\pi / 2$ is disfavored at more than $4 \sigma$ confidence level whereas for inverted mass ordering (IO) it is more stringent, where $\delta=\pi / 2$ is ruled out at more than $6 \sigma$. The best fit value of $\delta$ in NO and IO is near $1.2 \pi$ and $1.5 \pi$, respectively. For the sake of simplicity we work with only the best fit values. We have also estimated the highest and lowest values of the $\mathrm{CP}$ violating measure, the Jarlskog invariant $\left(J_{C P}=\right.$ 
TABLE 1: Input data from neutrino oscillation experiments [1].

\begin{tabular}{lcccc}
\hline Quantity & best fit values & $3 \sigma$ ranges & $2 \sigma$ ranges & $1 \sigma$ ranges \\
\hline$\Delta m_{21}^{2}\left[10^{-5} \mathrm{eV}^{2}\right]$ & 7.55 & $7.05-8.14$ & $7.20-7.94$ & $7.39-7.55$ \\
$\left|\Delta m_{31}^{2}\right|\left[10^{-3} \mathrm{eV}^{2}\right](\mathrm{NO})$ & 2.50 & $2.41-2.60$ & $2.44-2.57$ & $2.47-2.53$ \\
$\left|\Delta m_{31}^{2}\right|\left[10^{-3} \mathrm{eV}^{2}\right](\mathrm{IO})$ & 2.42 & $2.31-2.51$ & $2.34-2.47$ & $2.38-2.46$ \\
$\left.\theta_{12}\right|^{\circ}$ & 34.5 & $31.5-38.0$ & $32.2-36.8$ & $33.5-35.7$ \\
$\theta_{23} /^{\circ}(\mathrm{NO})$ & 47.7 & $41.8-50.7$ & $43.1-49.8$ & $46-48.9$ \\
$\theta_{23} /^{\circ}(\mathrm{IO})$ & 47.9 & $42.2-50.7$ & $44.5-48.9$ & $46.2-48.9$ \\
$\theta_{13} /^{\circ}(\mathrm{NO})$ & 8.45 & $8-8.9$ & $8.2-8.8$ & $8.31-8.61$ \\
$\theta_{13} /^{\circ}(\mathrm{IO})$ & 8.53 & $8.1-9$ & $8.3-8.8$ & $8.38-8.67$ \\
$\delta /^{\circ}(\mathrm{NO})$ & 218 & $157-349$ & $182-315$ & $191-256$ \\
$\delta /^{\circ}(\mathrm{IO})$ & 281 & $202-349$ & $229-328$ & $254-304$ \\
\hline
\end{tabular}

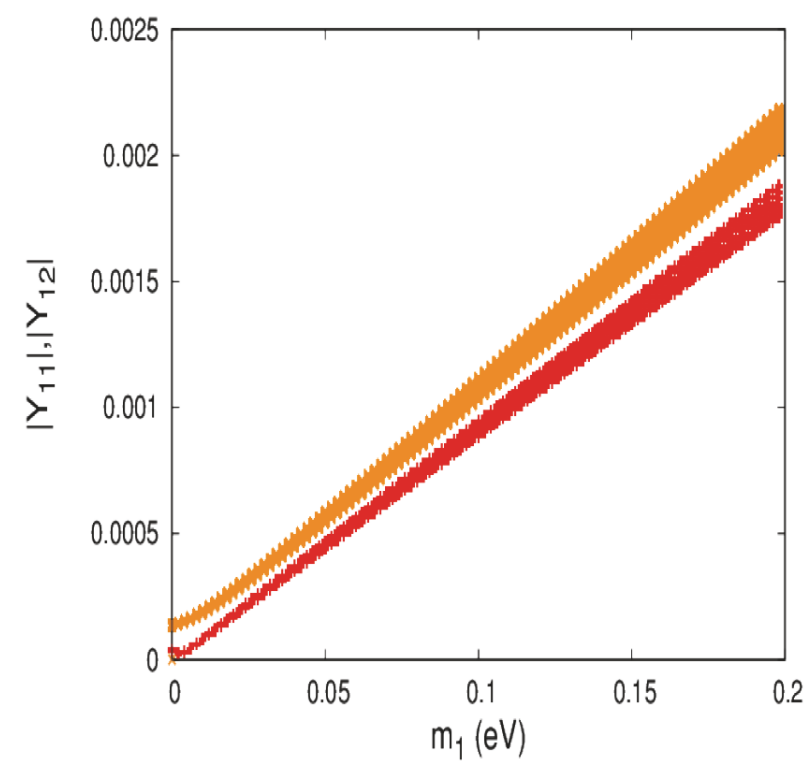

(a)

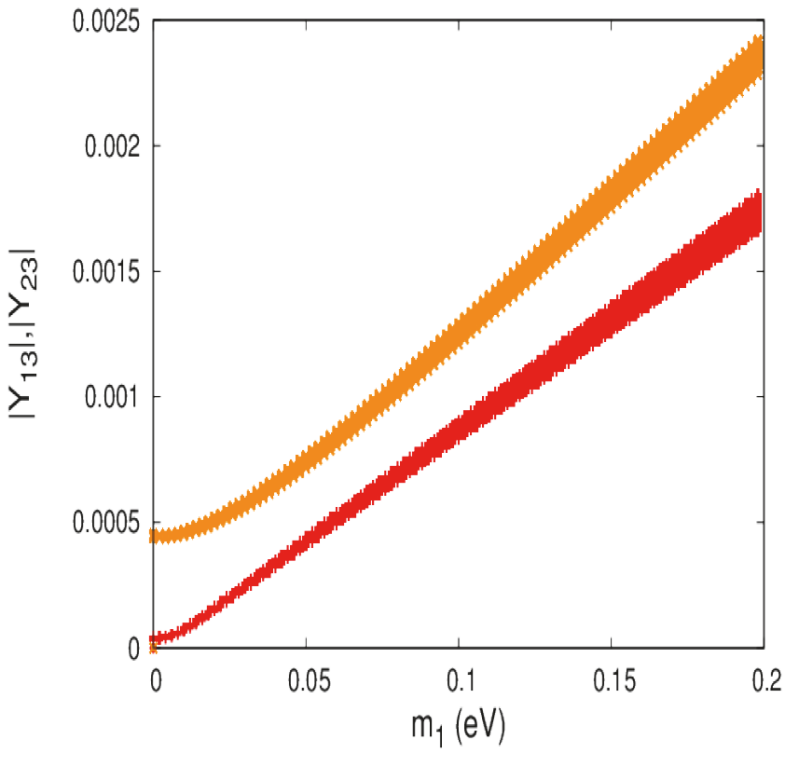

(b)

FIgURE 2: Determination of moduli of $Y$ matrix elements within $1 \sigma$ uncertainty of oscillation data as a function of lightest neutrino mass eigenvalues $m_{1}$. Phase angles used are randomly chosen Majorana phases $\alpha_{M}=37.91^{\circ}, \beta_{M}=157.91^{\circ}$ and central value of the Dirac phase $\delta=216^{\circ}$. In (a) red and yellow regions denote $1 \sigma$ allowed values $\left|Y_{11}\right|$ and $\left|Y_{12}\right|$, respectively. In (b) red patch gives values of $\left|Y_{13}\right|$ whereas yellow region denotes the same for $\left|Y_{23}\right|$ within the same uncertainty of the oscillation data.

$-s_{12} c_{12} s_{13} c_{13}^{2} s_{23} c_{23} \sin \delta$ ) for both the mass orderings. For NO: $J_{C P}=0.0175-0.0212$, for IO: $J_{C P}=0.0302-0.0365$ when $\delta$ is kept fixed at its best fit value whereas all other observables are varying in their respective $3 \sigma$ ranges.

2.1.3. Majorana Yukawa Coupling for $1 \sigma$ Bounds of Neutrino Oscillation Data. Here we follow exactly the same methodology as the previous case, however the numerical calculations are done with $1 \sigma$ ranges of oscillation data instead of $3 \sigma$ range. Here we are exploring the normally ordered case only. Unlike the previous case the lightest neutrino mass eigenvalue $m_{1}$ is not kept fixed; it is varied over a range of $(0-0.2) \mathrm{eV}$ and the corresponding variations of the modulus and phase of Majorana Yukawa couplings are depicted in Figures 2, 3, and 4. The $1 \sigma$ allowed ranges of those quantities for a fixed $m_{1}$ are also shown in Figures 5 and 6.

\section{Gauge Coupling Unification in the Scalar Extended SU(5)}

3.1. Lower Bound on the Scalar Triplet Mass. Exercising utmost economy in populating the grand desert, it was noted that the presence of the scalar component $\kappa(3,0,8) \subset 75_{H}$ at an intermediate mass $\simeq 10^{10} \mathrm{GeV}$ could achieve gauge coupling unification at $M_{G U T} \simeq 10^{15} \mathrm{GeV}$ [112] but no neutrino oscillation data was available at that time. Using the most recent electroweak precision data [111, 113, 114], in this work we find that this intermediate scalar mass is now reduced by one order, $M_{\kappa}=10^{9.23} \mathrm{GeV}$. Similarly the GUT scale is now determined with high precision including all possible theoretical and experimental uncertainties. Noting the result of this work as discussed in Section 2 that type-II seesaw realisation of neutrino mass needs $M_{\Delta}$ 


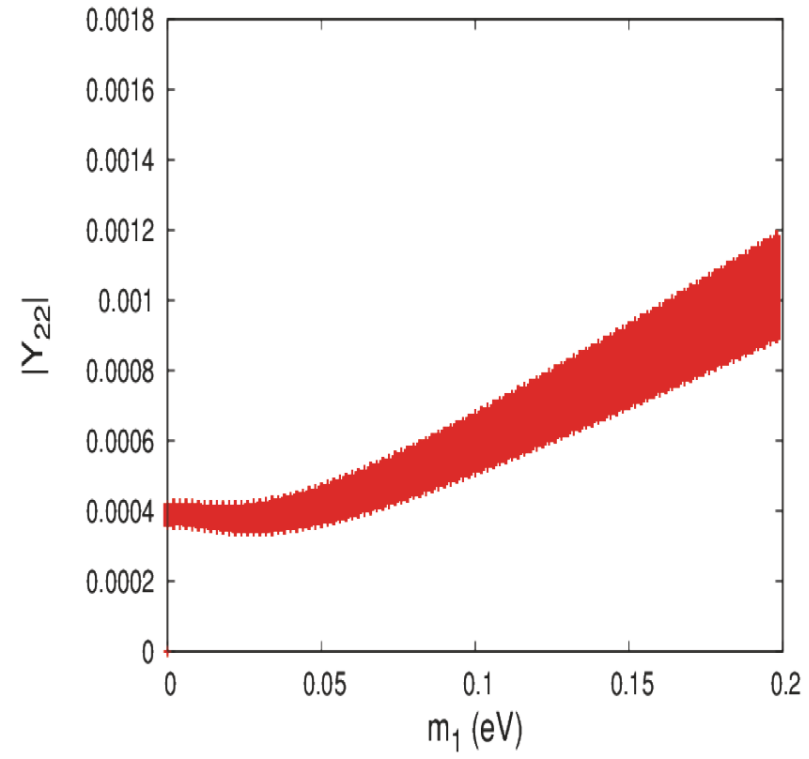

(a)

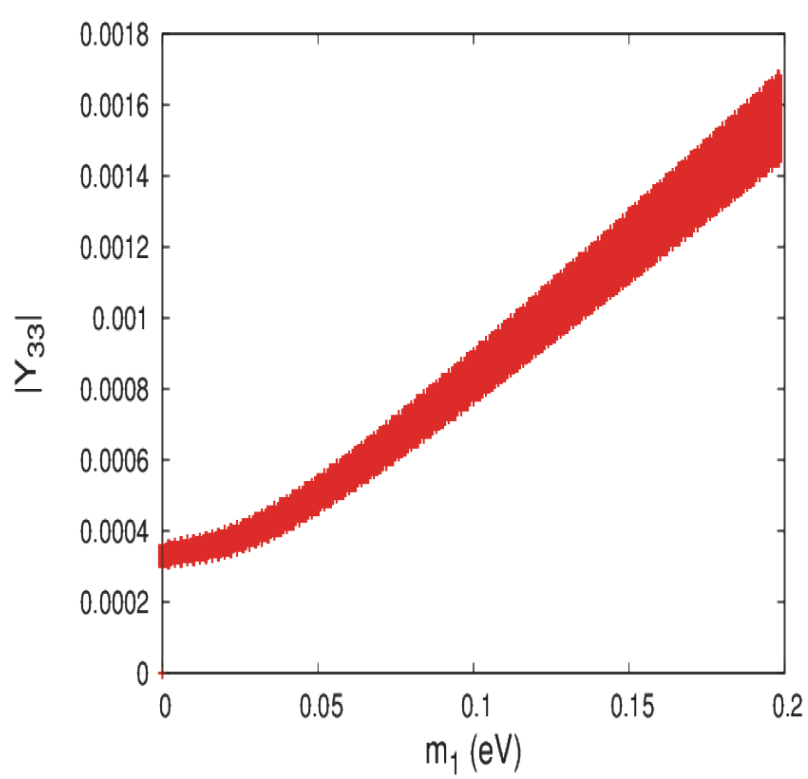

(b)

FIGURE 3: Determination of moduli of $Y$ matrix elements within $1 \sigma$ uncertainty of oscillation data as a function of lightest neutrino mass eigenvalues $m_{1}$ as shown in (a) for $\left|Y_{22}\right|$, and in (b) for $\left|Y_{33}\right|$. Phase angles used are the same as in Figure 2.

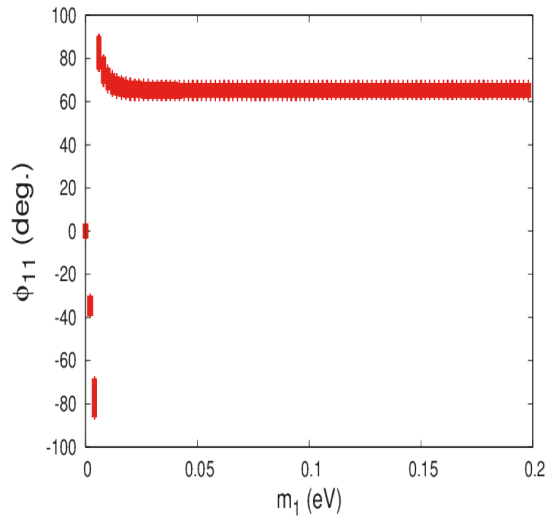

(a)

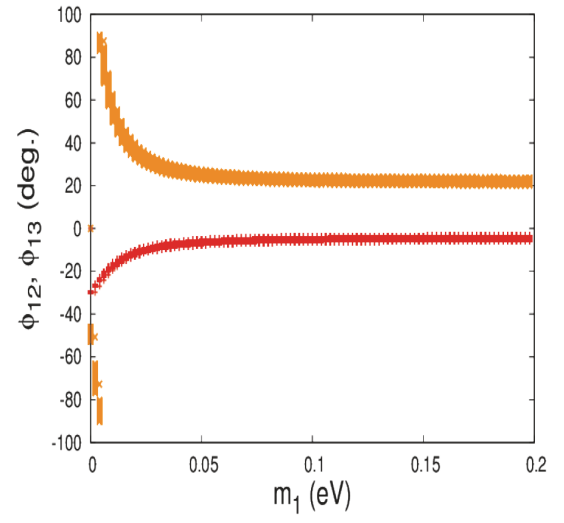

(b)

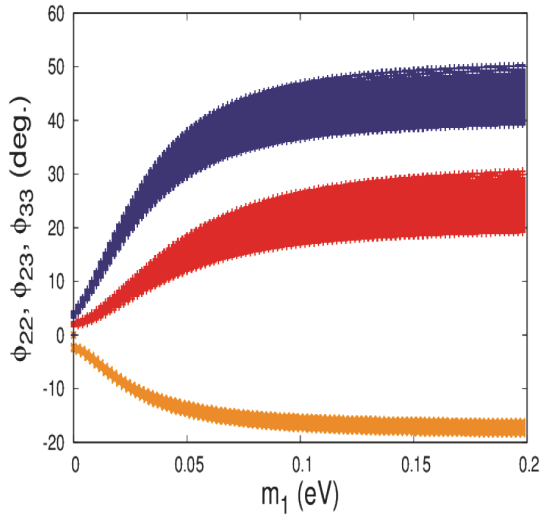

(c)

FIGURE 4: Determination of phases of $Y$ matrix elements as a function of lightest neutrino mass eigenvalues $m_{1}$ within $1 \sigma$ allowed uncertainty of oscillation data. Phase angles used are the same as in Figure 2. In (a) red region denotes values of $\phi_{11}$. In (b) red and yellow regions denote allowed values $\phi_{12}$ and $\phi_{13}$, respectively. In (c) red, yellow, and blue patches give allowed values of $\phi_{22}, \phi_{23}$, and $\phi_{33}$, respectively.

substantially lower than the GUT scale leads to the natural apprehension that the presence $\Delta_{L}(3,-1,1)$ at intermediate mass scale would destroy precision unification achieved by $\kappa(3,0,8)$. This apprehension is logically founded on the basis that nonvanishing contributions to the $S U(2)_{L}$ and $U(1)_{Y}$ beta functions would misalign the fine structure constants $\alpha_{2 L}(\mu)$ and $\alpha_{Y}(\mu)$ from the $\kappa(3,0,8)$ realised unification paths substantially for all mass scales $\mu>M_{\Delta}$.

We prevent any such deviation from the $\kappa(3,0,8)$ realisation of precision coupling unification by assuming all the components of $15_{H} \subset S U(5)$ to have the identical degenerate mass $M_{\Delta}$ which is bounded in the following manner:

$$
M_{\kappa} \leq\left(M_{\Delta}=M_{15_{H}}\right) \leq M_{\mathrm{GUT}} .
$$

Thus, in order to safeguard precision unification, it is essential that $M_{\Delta}=M_{15_{H}} \geq M_{\kappa}$ in the present scalar extended SU(5) model (the upper limit is due to our observation that typeII seesaw scale is lower than the GUT scale although, strictly speaking, $M_{\Delta}=M_{15_{H}}>M_{\mathrm{GUT}}$ is possible if type-II seesaw contribution to neutrino mass is ignored).

Thus type-II seesaw realisation of neutrino mass and precision unification in $\mathrm{SU}(5)$ needs the additional scalar representations $15_{H}$ and $75_{H}$.

3.2. RG Solutions to Mass Scales. For realistic unification of gauge couplings we use one loop equations [115] 


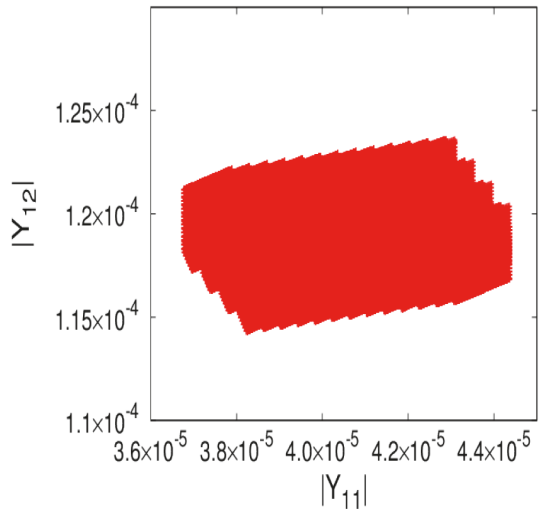

(a)

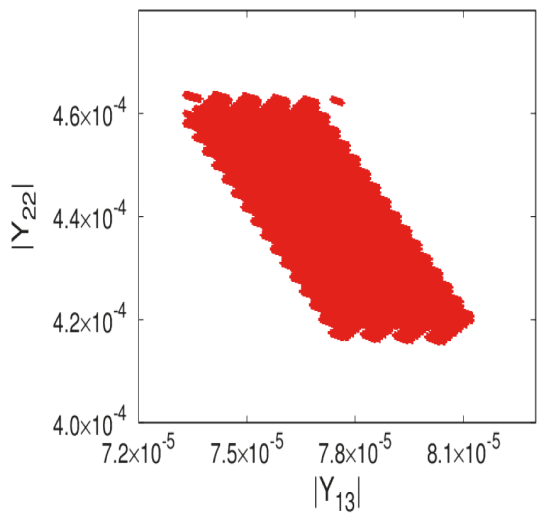

(b)

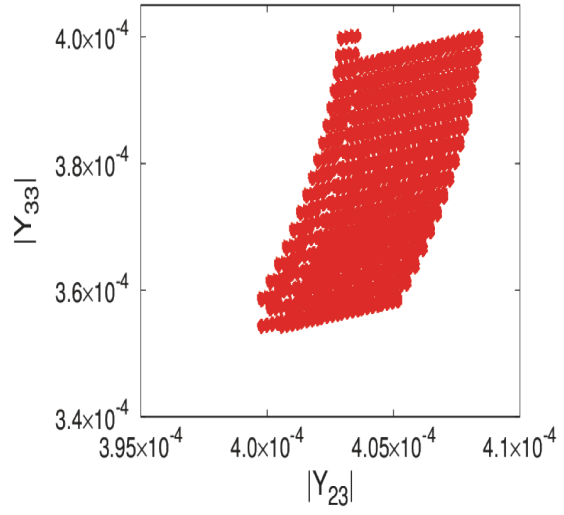

(c)

FiguRE 5: Determination of moduli of $Y$ matrix elements for a fixed value of lightest mass eigenvalue $m_{1}=0.00127 \mathrm{eV}$. Phase angles used for computation are $\alpha_{M}=124.37^{\circ}, \beta_{M}=86.27^{\circ}$ (randomly chosen), and $\delta=216^{\circ}$. Neutrino oscillation observables are varied within $1 \sigma$ range. (a) Variation of $\left|Y_{11}\right|$ with $\left|Y_{12}\right|$, (b) $\left|Y_{13}\right|$ versus $\left|Y_{22}\right|$, and (c) $\left|Y_{23}\right|$ versus $\left|Y_{33}\right|$.

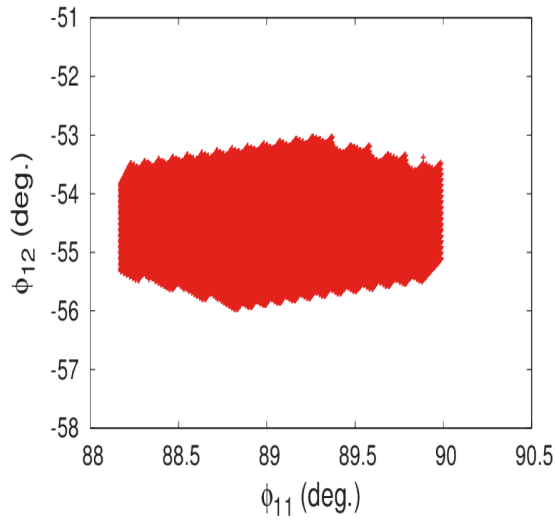

(a)

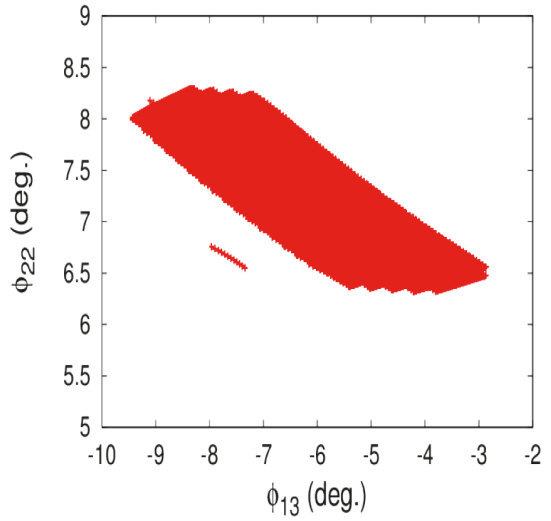

(b)

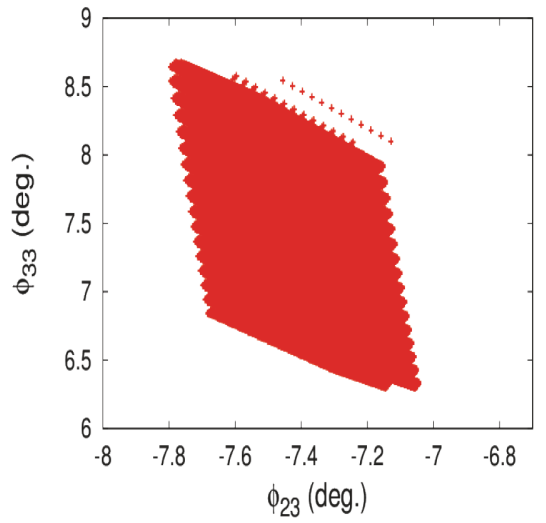

(c)

Figure 6: Determination of phases of $Y$ matrix elements for fixed value of lightest mass eigenvalue $m_{1}=0.001 \mathrm{eV}$. Phase angles used for computation are the same as in Figure 5. Neutrino oscillation observables are varied within $1 \sigma$ range; (a) variation of $\phi_{11}$ with $\phi_{12}$, (b) $\phi_{13}$ versus $\phi_{22}$, and (c) $\phi_{23}$ versus $\phi_{33}$.

supplemented by top-quark threshold effects [83] and twoloop corrections [116]

$$
\mu \frac{\partial g_{i}(\mu)}{\partial \mu}=\frac{a_{i}}{16 \pi^{2}} g_{i}^{3}+\frac{1}{\left(16 \pi^{2}\right)^{2}}\left[\Sigma_{j} b_{i j} g_{i}^{3} g_{j}^{2}-\kappa_{i} y_{\mathrm{top}}^{2}\right]
$$

In the range of mass scale $\mu=M_{Z}-M_{\mathrm{U}}$ we include top-quark Yukawa coupling $\left(y_{\text {top }}\right)$ contribution at the twoloop level with the coefficients in (14) $\kappa_{1 Y}=17 / 10, \kappa_{2 L}=$ $3 / 2, \kappa_{3 C}=2$ and the RG evolution equation [83]

$$
\mu \frac{\partial y_{t o p}}{\partial \mu}=\frac{y_{t o p}}{16 \pi^{2}}\left(\frac{9}{2} y_{t o p}^{2}-\frac{17}{20} g_{1 Y}^{2}-\frac{9}{4} g_{2 L}^{2}-8 g_{3 C}^{2}\right)
$$

The beta function coefficients in three different mass ranges $\mu=M_{Z} \longrightarrow M_{\kappa}, \mu=M_{\sigma}-M_{\Delta}$, and $\mu=M_{\Delta}-M_{U}$ are

$$
\mu=M_{Z} \longrightarrow M_{\kappa}:
$$

$$
\begin{gathered}
a_{Y}=\frac{41}{10}, \\
a_{2 L}=-\frac{19}{6}, \\
a_{3 C}=-7,
\end{gathered}
$$

$$
\underline{\mu}=M_{\kappa} \longrightarrow M_{\Delta}:
$$

$$
\begin{aligned}
& a_{Y}^{\prime}=\frac{41}{10}, \\
& a_{2 L}^{\prime}=-\frac{1}{2}, \\
& a_{3 C}^{\prime}=-\frac{11}{2},
\end{aligned}
$$


TABLE 2: Numerical values of the moduli $\left(\left|Y_{i j}\right|\right)$ and phases $\left(\phi_{i j}\right)(i, j=1,2,3)$ of Yukawa coupling matrix for normally ordered (NO) light neutrino masses corresponding to $3 \sigma$ global fit of neutrino oscillation data. Lightest neutrino mass eigenvalue is kept fixed at $m_{1}=0.00127$ $\mathrm{eV}$ for the sake of simplicity. Randomly chosen Majorana phases $\alpha_{M}=74.84^{\circ}, \beta_{M}=112.85^{\circ}$ and the central value of the Dirac phase $\delta=218^{\circ}$ have been used.

\begin{tabular}{lccccc}
\hline$\left|Y_{11}\right|$ & $\left|Y_{12}\right|$ & $\left|Y_{13}\right|$ & $\left|Y_{22}\right|$ & $\left|Y_{23}\right|$ & $\left|Y_{33}\right|$ \\
\hline$(1.74-3.95) \times 10^{-5}$ & $(1.13-1.44) \times 10^{-4}$ & $(4.09-6.71) \times 10^{-5}$ & $(3.20-4.67) \times 10^{-4}$ & $(4.07-4.35) \times 10^{-4}$ & $(3.05-4.5) \times 10^{-4}$ \\
$\phi_{11}$ & $\phi_{12}$ & $\phi_{13}$ & $\phi_{22}$ & $\phi_{23}$ & $\phi_{33}$ \\
$($ deg. $)$ & $($ deg. $)$ & $($ deg. $)$ & $($ deg. $)$ & $($ deg. $)$ & $($ deg. $)$ \\
$(-65.24)-(-61.73)$ & $(-48.50)-(-44.22)$ & $(-17.48)-8.27$ & $4.67-10.6$ & $(-6.81)-(-5.34)$ & $3.77-10.0$ \\
\hline
\end{tabular}

TABLE 3: Numerical values of the moduli $\left(\left|Y_{i j}\right|\right)$ and phases $\left(\phi_{i j}\right)(i, j=1,2,3)$ of Yukawa coupling matrix $Y$ for invertedly ordered (IO) light neutrino masses corresponding to $3 \sigma$ global fit of neutrino oscillation data. Lightest neutrino mass eigenvalue is kept fixed at $m_{3}=0.00127$ $\mathrm{eV}$. Phase angles used are the same as in Table 2.

\begin{tabular}{lccccc}
\hline$\left|Y_{11}\right|$ & $\left|Y_{12}\right|$ & $\left|Y_{13}\right|$ & $\left|Y_{22}\right|$ & $\left|Y_{23}\right|$ & $\left|Y_{33}\right|$ \\
\hline$(4.38-5.3) \times 10^{-4}$ & $(4.29-5.5) \times 10^{-4}$ & $(3.55-4.87) \times 10^{-4}$ & $(8.83-23.5) \times 10^{-5}$ & $(2.13-2.89) \times 10^{-4}$ & $(2.84-4.0) \times 10^{-4}$ \\
$\phi_{11}$ & $\phi_{12}$ & $\phi_{13}$ & $\phi_{22}$ & $\phi_{23}$ & $\phi_{33}$ \\
$($ deg. $)$ & $($ deg. $)$ & $($ deg. $)$ & $($ deg. $)$ & $($ deg. $)$ & $($ deg. $)$ \\
52.96 & $(-6.51)-(-3.16)$ & 0.5 & $(-60)-(-32.31)$ & $(-69.16)-(-51.39)$ & $(-78.89)-(-61.92)$ \\
68.35 & & 4.5 & & & \\
\hline
\end{tabular}

$\underline{\mu=M_{\Delta} \longrightarrow M_{U}:}$

$$
\begin{aligned}
& a_{Y}^{\prime \prime}=\frac{79}{15}, \\
& a_{2 L}^{\prime \prime}=\frac{2}{3}, \\
& a_{3 C}^{\prime \prime}=-\frac{13}{3} .
\end{aligned}
$$

We have used the most recent electroweak precision data [114]

$$
\begin{aligned}
\alpha_{S}\left(M_{Z}\right) & =0.1182 \pm 0.0005 \\
\sin ^{2} \theta_{W}\left(M_{Z}\right) & =0.23129 \pm 0.00005 \\
\alpha^{-1}\left(M_{Z}\right) & =127.94 \pm 0.02
\end{aligned}
$$

Using RGEs and the combinations $1 / \alpha\left(M_{Z}\right) \quad-$ $(8 / 3) 1 / \alpha_{2 L}\left(M_{Z}\right)$ and $1 / \alpha\left(M_{Z}\right)-(8 / 3) 1 / \alpha_{3 C}\left(M_{Z}\right)$, we have derived analytic formulas for the unification scale and intermediate $\operatorname{scale}\left(M_{\kappa}\right)$ treating $S U(2)_{L}$ triplet scalar scale $\left(M_{\Delta}\right)$ constant as

$$
\begin{aligned}
\ln \frac{M_{U}^{0}}{M_{Z}}= & \frac{2 \pi}{187 \alpha}\left(7-\frac{80 \alpha}{3 \alpha_{3 C}}+8 s_{W}^{2}\right)+\Delta_{U} \\
\ln \frac{M_{\kappa}^{0}}{M_{Z}}= & \frac{12 \pi}{187 \alpha}\left(5+\frac{23 \alpha}{3 \alpha_{3 C}}-21 s_{W}^{2}\right)+\Delta_{\kappa} \\
\frac{1}{\alpha_{G^{0}}}= & \frac{3}{8 \alpha}+\frac{1}{187 \alpha}\left(\frac{347}{8}+\frac{466 \alpha}{3 \alpha_{3 C}}-271 s_{W}^{2}\right) \\
& +\Delta_{\alpha_{G}}
\end{aligned}
$$

where $s_{W}^{2}=\sin ^{2} \theta_{W}\left(M_{Z}\right)$ and the first term in (20) represent one loop contributions. The terms $\Delta_{I}^{i}, i=U, \kappa, \alpha_{G}$, denote the threshold corrections due to unification scale $\left(M_{U}\right)$, intermediate scale $\left(M_{\kappa}\right)$, and GUT fine structure constant $\left(1 / \alpha_{G}\right)$.

Excellent unification of gauge couplings is found for

$$
\begin{aligned}
M_{U}^{0} & =10^{15.2+0.0312} \mathrm{GeV}, \\
M_{\kappa}^{0} & =10^{9.23} \mathrm{GeV}, \\
\alpha_{G_{0}}^{-1} & =41.79
\end{aligned}
$$

where the number 0.0312 in the exponent is due to GUT scale matching of inverse fine structure constant that is present even if all superheavy masses are exactly at $\mu=M_{U}^{0}[101,103-$ 105].

3.3. Effects of $15_{H}$ on Unification. It is well known that when a complete GUT representation is superimposed on an already realised unification pattern in non-SUSY GUTs $[117,118]$, the GUT scale is unchanged but the inverse fine structure constants change their slopes and deviate from the original paths proportionately so as to increase the unification coupling. As an example in non-SUSY $\mathrm{SO}(10)[117,118]$, at first a precision unification frame has been achieved with the modification of the TeV scale spectrum of the minimal SUSY GUT by taking out the full scalar super partner content of the spinorial super field representation $16 \subset S O(10)$. Then the resulting $\mathrm{TeV}$ scale spectrum is $[117,118]$

$$
\begin{aligned}
& \chi\left(2,-\frac{1}{2}, 1\right), \\
& F_{\phi}\left(2, \frac{1}{2}, 1\right),
\end{aligned}
$$




$$
\begin{gathered}
F_{\chi}\left(2,-\frac{1}{2}, 1\right), \\
F_{\sigma}(3,0,1), \\
F_{b}(1,0,1), \\
F_{c}(1,0,8)
\end{gathered}
$$

which may be recognised to be the same as the corresponding spectrum in the split-SUSY case supplemented by the additional scalar doublet $\chi(2,-1 / 2,1)$. In $3.3 F_{i}$ 's represent nonstandard fermions. Further adjustment of masses of these particles around $\mathrm{TeV}$ scale has been noted to achieve degree of precision coupling unification higher than MSSM [117]. After having thus achieved a precision unification, the full $15_{H}$ is superimposed at the type-II seesaw scale $M_{\Delta}$ of the nonsupersymmetrised unification framework. Analogous to MSSM, this model [118] predicts a number of fermions as in 3.3 at the $\mathrm{TeV}$ scale which must be verified experimentally at accelerator energies.

In contrast, the present model has only the standard Higgs doublet $\phi(2,1 / 2,1)$ and the WIMP DM scalar singlet $\xi(1,0,1)$ near $\mathrm{TeV}$ scale as discussed below. Although the $\mathrm{TeV}$ scale DM has not been confirmed yet by direct experiments, LUX16 or Femi-LAT-like experiments may detect it. Moreover, as shown below, the present model ensures vacuum stability through this WIMP dark matter candidate whereas in [118] the vacuum stability and DM issues are yet to be answered. Further, the SM coupling unification scale in [118] being close to the SUSY GUT scale, $M_{U} \sim 10^{16} \mathrm{GeV}$, predicts proton lifetime nearly 60 times larger than the current experimental limit without threshold effect which is expected to introduce larger uncertainty compared to the present model. It may be more difficult to verify this model by ongoing proton decay experiments. But the present model including such uncertainties is within the experimentally accessible limits. The origin of invariant GUT scale in the presence of $15_{H}$ in the present model is due to the invariance of the beta function differences which is $-7 / 6$ in this model

$$
\Delta a_{i}=\left(a_{i}^{\prime}-a_{i}^{\prime \prime}\right)=-\left(\frac{7}{6}\right), \quad(i=1,2,3) .
$$

This results in a change in the inverse GUT coupling constant $\alpha_{G}^{-1}$ which occurs due to the RG predicted modification

$$
\begin{aligned}
\frac{1}{\alpha_{G}}= & \frac{1}{\alpha_{G^{0}}}-\frac{1}{561 \alpha}\left(\frac{229}{2}+\frac{134 \alpha}{3 \alpha_{3 C}}-350 s_{W}^{2}\right) \\
& +\frac{7}{12 \pi} \ln \left(\frac{M_{\Delta}}{M_{Z}}\right) .
\end{aligned}
$$

Thus the result of type-II seesaw motivated insertion of $15_{H}$ into the $\kappa$-realised unification framework is to decrease in the inverse GUT fine structure constant while keeping mass scales same as in (21)"

$$
\alpha_{G}^{-1}=37.765 .
$$

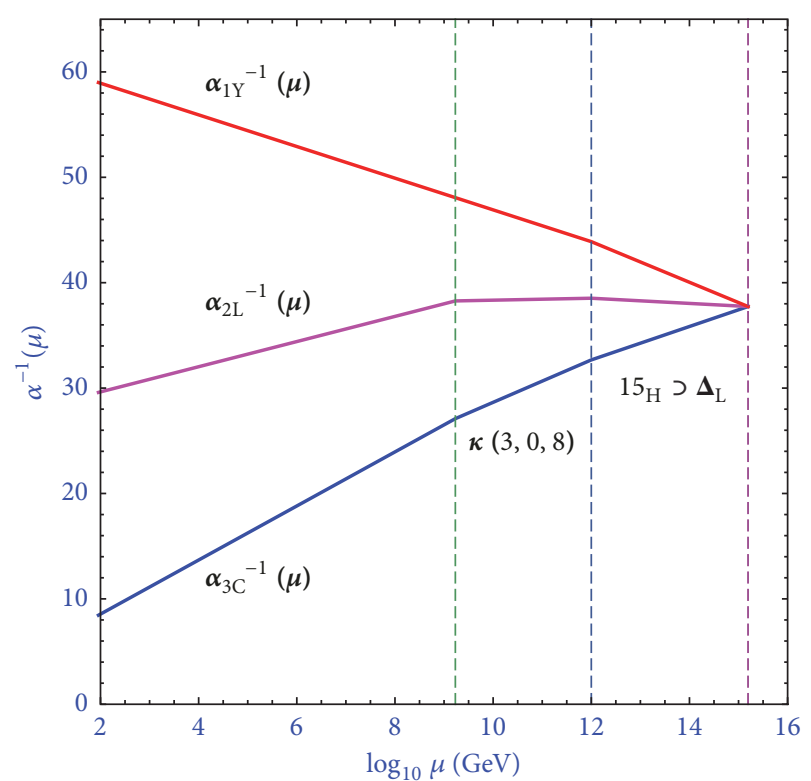

FIGURE 7: Unification of SM gauge couplings in the presence of $\kappa(3,0,8)$ at $M_{\kappa}=10^{9.23} \mathrm{GeV}$ and $15_{H} \subset S U(5)$ at $M_{15}=10^{12}$ $\mathrm{GeV}$ as discussed in the text. The vertical dashed lines represent the intermediate scale masses and GUT scales.

which is a $9.6 \%$ effect. It is essential to take this effect into consideration in the top-down approach for consistency with the precision value of the electromagnetic fine structure constant $\alpha^{-1}\left(M_{Z}\right)=127.9 \pm 0.01$ [114]. More important is its visible effect on proton lifetime prediction. It is clear from $\alpha_{G}^{-2}$ dependence in (36) of Section 4, (21), and (25) that the this intermediate type-II seesaw scale has a proton lifetime reduction by $19 \%$ that further reduces for lower seesaw scales, $M_{\Delta}<10^{12} \mathrm{GeV}$. But the reduction effect decreases as $M_{\Delta}$ increases such that the proton lifetime remains unchanged for the limiting value $M_{\Delta}=M_{15_{H}}=M_{U}$.

In Figure 7 we have shown evolution of inverse fine structure constants of three gauge couplings of SM against mass scales depicting precision unification at $M_{U}^{0}=10^{15.2}$ $\mathrm{GeV}$.

3.3.1. Implications for Lepton Number and Flavor Violations. It is evident from (13) and (21) that the numerical lower bound on the masses of three members of the triplet in $\Delta_{L}(3,-1,1)$ is

$$
M_{\Delta^{0}} \simeq M_{\Delta^{-}} \simeq M_{\Delta^{--}} \geq 10^{9.23} \mathrm{GeV}
$$

Out of these we have discussed in Section 2 how the mediation of $M_{\Delta^{0}}$ gives type-II seesaw contribution to neutrino masses matching with available neutrino oscillation data at $1 \sigma-3 \sigma$ levels for all types of hierarchies: $\mathrm{NH}, \mathrm{IH}$, and QD. As a result the Higgs-Yukwa interaction and induced VEV 
of the neutral component of the triplet in the present SU(5) model give similar predictions as in the triplet extended SM based analyses [119] or in the left-right symmetric models and $\mathrm{SO}(10)$ with large $W_{R}$ boson mass $[45,120]$. Currently a number of experimental investigations are underway to detect the double beta decay process that would establish Majorana nature of neutrino. The most important difference from such SM based phenomenological analyses is that in the present $\mathrm{SU}(5)$ model with type-II seesaw all the parameters of the neutrino oscillation data are theoretically predicted by the seesaw mechanism. Even though $M_{\Delta^{0}} \geq 10^{9.23} \mathrm{GeV}$, it predicts the double beta decay lifetime close to the observable limit of $\tau_{\beta \beta} \sim 5 \times 10^{25}$ yrs for QD type light neutrino masses $\widehat{m}_{i} \sim 0.2 \mathrm{eV}$. On the other hand for $\mathrm{NH}$ type of hierarchy the predicted decay rate is much lower with lifetime $\tau_{\beta \beta} \gg 10^{29}$ yrs. Another theoretical contribution to the double beta decay process is due to the mediation of the doubly charged component $\Delta^{--}$through the physical process $W^{-} W^{-} \longrightarrow \Delta^{--} \longrightarrow e^{-} e^{-}$which is negligible because of additional damping of the amplitude caused by the inverse square of its heavy mass $M_{\Delta^{--}} \geq 10^{9.23} \mathrm{GeV}$. The charged component $\Delta^{-}$also mediates a new loop contributions to lepton flavor violating processes such as $l_{\alpha} \longrightarrow l_{\beta}+\gamma$. Again, because of heavy triplet mass the respective contribution to branching ratio turns out to be much smaller than the corresponding prediction with $S M$ (supplemented by the oscillation data): $B r .(\mu \longrightarrow e \gamma)<10^{-53}[45,120]$. Similarly the tree level mediation of the LFV process $\mu \longrightarrow$ eee by $\Delta^{--}$ is severely damped out compared to the loop mediated $W-$ boson contribution.

3.4. Threshold Effects on the GUT Scale. In the single step breaking model discussed in this work, GUT threshold effects due to superheavy degrees of freedom in different SU(5) representations are expected as major sources of uncertainties on unification scale and proton lifetime prediction. We have estimated the threshold uncertainties following the partially degenerate assumption introduced in [121, 122] which states that the superheavy components belonging to the same GUT representation are degenerate with a single mass scale.

The analytic formulas for GUT threshold effects on the unification scale, intermediate scale, and GUT fine structure constant are

$$
\begin{aligned}
\Delta_{I}^{\kappa} & =\Delta \ln \frac{M_{\kappa}}{M_{Z}}=\frac{1}{2244}\left(123 \lambda_{2 L}-215 \lambda_{Y}+92 \lambda_{3 C}\right), \\
\Delta_{I}^{U} & =\Delta \ln \frac{M_{U}}{M_{Z}}=\frac{5}{3366}\left(3 \lambda_{2 L}+13 \lambda_{Y}-16 \lambda_{3 C}\right), \\
\Delta_{I}^{\alpha_{G}} & =\Delta\left(\frac{1}{\alpha_{G}}\right) \\
& =\frac{1}{80784 \pi}\left(-948 \lambda_{2 L}-2425 \lambda_{Y}+5056 \lambda_{3 C}\right) .
\end{aligned}
$$

In (27) $\lambda_{i}, i=2 L, Y, 3 C$ are matching functions due to superheavy scalars $(S)$ and gauge bosons $(V)$ to the three gauge couplings,

$$
\begin{aligned}
\alpha_{i}^{-1}\left(M_{U}\right) & =\alpha_{G}^{-1}-\frac{\lambda_{i}\left(M_{U}\right)}{12 \pi}, \\
\lambda_{i}^{S}\left(M_{U}\right) & =\sum_{j} \operatorname{Tr}\left(t_{i S j}^{2} \widehat{p}_{S j} \ln \frac{M_{j}^{S}}{M_{U}}\right), \\
\lambda_{i}^{V}\left(M_{U}\right) & =\sum_{l} \operatorname{Tr}\left(t_{i V l}^{2}\right)-21 \sum_{l} \operatorname{Tr}\left(t_{i V l}^{2} \ln \frac{M_{l}^{V}}{M_{U}}\right),
\end{aligned}
$$

where $t_{i S}$ and $t_{i V}$ represent the matrix representations due to broken generators of scalars and gauge bosons. The term $\widehat{p}_{S j}$ denotes the projection operator that removes the Goldstone components from the scalars contributing to spontaneous symmetry breaking.

The decomposition of different SU(5) representations under $G_{213}$ with respect to their superheavy components and values of corresponding matching functions is presented in Table 4.

Using the values of matching function $\lambda^{i}\left(M_{U}\right)$ from Table 4 in (27) we estimate corrections to different mass scales due to superheavy masses as

$$
\begin{aligned}
\Delta \ln \frac{M_{\kappa}}{M_{Z}}= & 0.0026738 \eta_{5}+0.23262 \eta_{24}-1.24599 \eta_{75} \\
\Delta \ln \frac{M_{U}}{M_{Z}}= & -0.0160428 \eta_{5}-0.0623886 \eta_{24} \\
& +1.142602 \eta_{75} \\
\Delta\left(\frac{1}{\alpha_{G}}\right)= & 0.0160999 \eta_{5}+0.0522951 \eta_{24} \\
& +0.0462547 \eta_{75} .
\end{aligned}
$$

Maximising the uncertainty in $M_{U}$ leads to

$$
\begin{aligned}
\Delta \ln \left(\frac{M_{U}}{M_{Z}}\right) & = \pm 0.22103 \eta_{S H}, \\
\Delta \ln \left(\frac{M_{\kappa}}{M_{Z}}\right) & = \pm 1.48128 \eta_{S H}, \\
\Delta\left(\frac{1}{\alpha_{G}}\right) & = \pm 0.02214 \eta_{S H},
\end{aligned}
$$

where $\eta_{S H}=\ln \left(M_{S H} / M_{U}\right)$ and $M_{S H} / M_{U}=n(1 / n)$ with plausible allowed values of real number $n=1-10$.

We also note that the degenerate superheavy gauge bosons contribute a significant correction to unification scale

$$
\left(\frac{M_{U}}{M_{U}^{0}}\right)_{V}=10^{ \pm 0.65508}
$$

Adding all corrections together we obtain

$$
M_{U}=10^{15.2312 \pm 0.11 \pm 0.221 \eta_{S} \pm 0.655 \eta_{V}} \mathrm{GeV} \text {. }
$$

The first uncertainty $( \pm 0.11)$ represents uncertainty in input parameters given in (19). 
TABLE 4: Superheavy components of SU(5) representations under the SM gauge group $G_{213}$ used to estimate GUT threshold effects.

\begin{tabular}{|c|c|c|}
\hline SU(5)representations & $G_{213}$ submultiplet & $\left(\lambda_{2 L}, \lambda_{1 Y}, \lambda_{3 C}\right)$ \\
\hline $5_{H}$ & $C_{1}(1,-1 / 3,3)$ & $\left(0, \frac{2}{3}, 1\right)$ \\
\hline \multirow{2}{*}{$24_{H}$} & $D_{1}(3,0,1)$ & $(2,0,0)$ \\
\hline & $D_{2}(1,0,8)$ & $(0,0,3)$ \\
\hline \multirow{7}{*}{$75_{H}$} & $E_{1}(1,10 / 3,3)$ & $\left(0,5, \frac{1}{2}\right.$ \\
\hline & $E_{2}(2,5 / 3,3)$ & $\left(\frac{3}{2}, \frac{5}{2}, 1\right.$ \\
\hline & $E_{3}(1,-10 / 3, \overline{3})$ & $\left(0,5, \frac{1}{2}\right.$ \\
\hline & $E_{4}(2,-5 / 3, \overline{3})$ & $\left(\frac{3}{2}, \frac{5}{2}, 1\right)$ \\
\hline & $E_{5}(2,-5 / 3, \overline{6})$ & $(3,5,5)$ \\
\hline & $E_{6}(2,5 / 3,6)$ & $(3,5,5)$ \\
\hline & $E_{7}(1,0,8)$ & $(0,0,3)$ \\
\hline \multirow{3}{*}{$15_{H}$} & $\Delta_{L}(3,-1,1)$ & $\left(4, \frac{18}{5}\right.$ \\
\hline & $H_{2}(2,1 / 6,3)$ & \\
\hline & $H_{3}(1,2 / 3,6)$ & $0, \frac{16}{5}, 5$ \\
\hline $24_{V}$ & $\begin{array}{c}V_{1}\left(2,-\frac{5}{6}, 3\right) \\
V_{2}\left(2, \frac{5}{6}, \overline{3}\right)\end{array}$ & $\left(\begin{array}{l}\left.\frac{3}{4}, \frac{5}{4}, \frac{1}{2}\right) \\
\left(\frac{3}{4}, \frac{5}{4}, \frac{1}{2}\right)\end{array}\right.$ \\
\hline
\end{tabular}

\section{Proton Lifetime Prediction}

Currently the measured value on the lower limit of the proton lifetime for the decay mode $p \longrightarrow e^{+} \pi^{0}$ is $[109,123-126]$

$$
\tau_{p}^{\text {expt. }} \geq 1.6 \times 10^{34} \mathrm{yrs}
$$

Including strong and electroweak renormalization effects on the $d=6$ operator and taking into account quark mixing, chiral symmetry breaking effects, and lattice gauge theory estimations, the decay rates are $[37,127,128]$

$$
\begin{aligned}
& \Gamma\left(p \longrightarrow e^{+} \pi^{0}\right) \\
& \quad=\left(\frac{m_{p}}{64 \pi f_{\pi}^{2}} \frac{\alpha_{G}{ }^{4}}{M_{U}{ }^{4}}\right)\left|A_{L}\right|^{2}\left|\overline{\alpha_{H}}\right|^{2}\left(1+D^{\prime}+F\right)^{2} \times R,
\end{aligned}
$$

where $R=\left[A_{S R}^{2}+A_{S L}^{2}\left(1+\left|V_{u d}\right|^{2}\right)^{2}\right]$ for $S U(5), V_{u d}=$ $0.974=$ the $(1,1)$ element of $V_{C K M}$ for quark mixings, and $A_{S L}\left(A_{S R}\right)$ is the short-distance renormalization factor in the left (right) sectors. In (35) $A_{L}=1.25=$ long distance renormalization factor but $A_{S L} \simeq A_{S R}=2.542$. These are numerically estimated by evolving the dim.6 operator for proton decay by using the anomalous dimensions of [129] and the beta function coefficients for gauge couplings of this model. In (35) $M_{U}=$ degenerate mass of superheavy gauge bosons, $\bar{\alpha}_{H}=$ hadronic matrix elements, $m_{p}=$ proton mass $=938.3 \mathrm{MeV}, f_{\pi}=$ pion decay constant $=139 \mathrm{MeV}$, and the chiral Lagrangian parameters are $D=0.81$ and $F=0.47$. With $\alpha_{H}=\overline{\alpha_{H}}\left(1+D^{\prime}+F\right)=0.012 \mathrm{GeV}^{3}$ estimated from lattice gauge theory computations [130-132], we obtain $A_{R} \simeq$
$A_{L} A_{S L} \simeq A_{L} A_{S R} \simeq 2.726$ and the expression for the inverse decay rate is

$$
\Gamma^{-1}\left(p \longrightarrow e^{+} \pi^{0}\right)=\frac{4}{\pi} \frac{f_{\pi}^{2}}{m_{p}} \frac{M_{U}^{4}}{\alpha_{G}^{2}} \frac{1}{\alpha_{H}^{2} A_{R}^{2}} \frac{1}{F_{q}}
$$

where the GUT fine structure constant $\alpha_{G}=0.0263$ and the factor $F_{q}=\left(1+\left(1+\left|V_{u d}\right|^{2}\right)^{2}\right) \simeq 4.8$. This formula has the same form as given in [127] which has been modified here for the SU(5) case. gives

Using the estimated values of the model parameters, (36)

$$
\tau_{p}^{S U(5)} \simeq 10^{33.110 \pm 0.440 \pm 0.884\left|\eta_{S}\right| \pm 2.62\left|\eta_{V}\right|} \text { yrs. }
$$

Numerical estimations on proton lifetime are shown in Table 5 for different splitting factors of superheavy masses.

It is interesting to note that despite three Higgs representations $5_{H}, 24_{H}, 75_{H}$, major contribution to threshold uncertainty in the model is only due to superheavy gauge bosons. When all superheavy gauge boson masses are identically equal to $M_{U}$, superheavy scalar mass splitting by a factor $20(1 / 20)$ from the GUT scale gives $\eta_{S}=1.3(-1.3)$ leading to $\left[\tau_{p}\right]_{\max }=1.80 \times 10^{34} \mathrm{yrs}$. which is consistent with the current experimental bound.

\section{Scalar Dark Matter in SU(5)}

5.1. Phenomenological and Experimental Constraints. The existence of dark matter (DM) in our galaxy has been established beyond any doubt through its gravitational effects by numerous observations [133]. Hence the hunt for DM has 
TABLE 5: Upper limits on predicted proton lifetime as a function of superheavy scalar (S) and gauge boson(V) mass splittings as defined in the text. The factor $10^{ \pm 0.44}$ represents uncertainty due to input parameters.

\begin{tabular}{lccccc}
\hline$M_{S}$ & $M_{V}$ & $\tau_{P}(y r s)$ & $\frac{M_{S}}{M_{U}}$ & $\frac{M_{V}}{M_{U}}$ & 5 \\
\hline 10 & $M_{U}$ & $9.77 \times 10^{33 \pm 0.44}$ & 5 & 6 & $3.59 \times 10^{35 \pm 0.44}$ \\
10 & 1 & $6.00 \times 10^{34 \pm 0.44}$ & 3 & 10 & $3.68 \times 10^{35 \pm 0.44}$ \\
8 & 2 & $1.42 \times 10^{35 \pm 0.44}$ & 1 & 1 & $5.32 \times 10^{35 \pm 0.44}$ \\
6 & 3 & $2.35 \times 10^{35 \pm 0.44}$ & 20 & $1.80 \times 10^{34 \pm 0.44}$ \\
\hline
\end{tabular}

been assumed to be of paramount importance for the particle physics community to understand its nature in particular and that of the universe in general. To this end, experiments using a wide range of approaches are being pursued worldwide and giving a large spectrum of interpretations of the DM candidates with masses ranging from a few $\mathrm{eV}$ to $\mathrm{PeV}$ or even beyond, from axions to wimpzillas and decaying dark matter.

Our motivation in this section is to explore whether $\mathrm{SU}(5)$ model can accommodate a scalar singlet $(=\xi)$ as a candidate DM which might be instrumental in contributing to the observed relic density or may be detected through ongoing direct or indirect search experiments. The local DM density is observed with some uncertainty to be $0.4 \mathrm{GeV} / \mathrm{cm}^{3}$ [134]. Earlier measurements by WMAP [8] and more recent observation by PLANCK satellite [29] indicate $85 \%$ of matter content of the Universe to be DM with its relic density

$$
\Omega_{\mathrm{dm}} h_{\text {Hubble }}^{2}=0.1198 \pm 0.0026
$$

where $h_{\text {Hubble }}$ is the Hubble parameter. Various attractive models have been proposed to explain the observed relic density of dark matter and its stability with half-life greater than the age of the universe, $\tau_{D M}>10^{17} \mathrm{~s}$. Attempts in this direction include addition of scalar or fermionic dark matter candidates to the $\mathrm{RH}$ neutrino $(\mathrm{RH} v)$ extended SM. Following the work of Lee-Weinberg [135] and in big-bang cosmology, a weakly interacting massive particle (WIMP) has enjoyed a special status as a DM candidate as it can naturally explain the observed relic density. Model independent upper bound on the WIMP DM mass has been also derived from perturbative unitarity [136] with $M_{\text {WIMP }} \leq 100 \mathrm{TeV}$. Recently extensive investigations have been made to explore possible special symmetries underlying the dynamics of DM [137139].

5.1.1. Direct Detection of Dark Matter. Since DM particles are electrically neutral and cosmologically stable, they are referred to as missing energy at colliders where searches for DM mainly focus on the detection of visible signals like jets and charged leptons. At colliders we can study DM either through investigating its direct detection signals or indirect detection signals. The scalar singlet DM in our model may be discovered through direct and indirect signals. In particular, XENON1T experiment may discover or rule out the scalar singlet DM for reasonable values of DM mass and Higgs portal coupling, rejecting its nonperturbative values higher than $1.5 \mathrm{TeV}[140,141]$.

Several terrestrial experiments like CDMS [142, 143], DAMA/NAI [144, 145], XEXNON100 [16], and LUX [18] are still going on around the globe for direct detection of dark matter. These underground detectors are constructed using various targets made up of $\mathrm{Xe}, \mathrm{Ge}, \mathrm{NaI}$, etc. in an attempt to explore either electronic or nuclear scatterings at low energies. In this case, the recoil energy is usually observed from the scattering between DM particles and nucleons [146] or from scattering between electrons and dark matter. The direct search experiments, XENON100 [16] and LUX, predict an upper bound in the $M_{D M}-\sigma_{D M}$ plane where $\sigma_{D M}$ represents DM elastic scattering cross section and $M_{D M}$ stands for DM mass. These experiments furnish very stringent bounds on dark matter-nucleon scattering cross section for different DM masses. For example, LUX and XENON100 experiments predict similar DM-nucleon cross section bound at around $10^{-44} \mathrm{~cm}^{2}$ for a DM mass of $1000 \mathrm{GeV}$ whereas XENON1T search predicts a smaller cross section bound $2 \times 10^{-46} \mathrm{~cm}^{2}$ for the same DM mass keeping the DM relic density in the right ballpark [147, 148]. A concise review of current status of scalar singlet dark matter is available in [149] where references to most of the recent experimental and phenomenological investigations are available. In general, for elastic scattering of a DM particle off nucleons, either a standard Higgs or a $Z$-boson exchange is needed in the $\mathrm{t}$-channel of the dominant tree diagrams. Even though the singlet scalar $\mathrm{DM} \xi(1,0,1)$ has no gauge interaction, still it can elastically scatter off nucleons in direct search experiments through Higgs exchange via quartic Higgs portal interaction

$$
V_{\text {Port }}=\frac{\lambda_{\phi \xi}}{2} \phi^{\dagger} \phi \xi^{2}+\text { h.c, }
$$

where the standard Higgs VEV and the portal quartic coupling $\lambda_{\phi \xi}$ contribute directly to the cross section in the lowest order.

Although till today no signals in direct detection experiments have been observed except for the controversial DAMA modulation signal, direct detection searches still have the potential to unravel the mystery of DM because of the fact that if a signal is observed, we can correlate the scattering cross section and mass of the DM particle with its local density.

5.1.2. Indirect Detection of Dark Matter. In indirect dark matter detection (IDMD) experiments, the DM particles may annihilate or decay to standard model particles or other exotic final states in a region of high DM density and finally manifest as a visible signal in form of gamma rays, cosmic rays, neutrinos, and positrons or antiparticles. Such events 
are expected to exhibit excesses over the desired abundance of the particles in the cosmos. The IDMD searches like Fermi-LAT [19], AMS [150], HESS [151], MAGIC [152], ATIC [153], DAMPE [154], PLANCK [29], ICECUBE [20, 21], etc. basically look for these excesses in the universe to confirm the detection of DM annihilation. For example, DM could be detected through the observation of neutrino fluxes by ICECUBE telescope arising from annihilation dark matter. The IceCube neutrino events have been recently interpreted to be consistent with decaying dark matter mass in the $\mathrm{PeV}$ range or larger.

Recently IDMD searches gave several hints for DM detection like lines at $3.5 \mathrm{KeV}[155,156], 130 \mathrm{GeV}[157$, $158]$, and the gamma ray excess from the galactic centre [159]. However, no conclusive and consistent information has emerged so far. These signals have been attributed to either astrophysical sources or instrumental effects $[160,161]$.

Recent data from LUX-2016 and Fermi-LAT $[18,19]$ have constrained the DM mass as well as its unknown Higgs portal coupling. It can be shown that $\lambda_{\phi \xi} \sim \mathcal{O}(0.01)$ can generate the right relic density with low mass $\xi$ of order $50 \mathrm{GeV}$. On the other hand direct DM searches from the LUX-16 data have ruled out the existence of scalar DM $\xi$ over a wider mass range $M_{\xi} \simeq 70-500 \mathrm{GeV}$. In summary, the scalar dark matter mass can be on the lower side

$$
M_{\xi}<60 \mathrm{GeV}
$$

contributing prominently to relic density, or on the higher side

$$
100 \mathrm{TeV}>M_{\xi}>500 \mathrm{GeV}
$$

In (41) the LHS is due to the perturbative unitarity bound [135] and the RHS is due to [18].

5.2. Embedding in $S U(5)$. Besides the $\mathrm{SU}(5)$ Higgs representations $5_{H}, 24_{H}, 15_{H}$, and $75_{H}$, we further extend its scalar sector by the scalar singlet DM $\xi(1,0,1)$ which we assume to be also a $\mathrm{SU}(5)$ singlet. Obviously it has no direct gauge boson interaction of any kind. But it has interaction with SM Higgs through Higgs portal of the type shown in (39). Then it can have gauge interaction in higher orders. In any theoretical model, the stability of DM must be ensured such that its lifetime is longer than the lifetime of the universe. Usually a discrete symmetry $Z_{2}$ is imposed to safeguard the stability.

We assign all the fermions in $\overline{5}_{F}, 10_{F}$, and consequently the SM fermions, to possess $Z_{2}=-1$. The Higgs representations $5_{H}, 24_{H}, 15_{H}$, and $75_{H}$ are assigned $Z_{2}=+1$. Needless to mention that the SM Higgs doublet $\phi, \kappa(3,0,8)$, and $\Delta_{L}(3,0,1)$ have the same value of $Z_{2}=+1$. Out of all the scalars only the DM singlet scalar is assigned odd value of $Z_{2}=-1$. This assignment prevents direct Yukawa interaction of $\xi$ and ensures its desired stability.

\section{Vacuum Stability in SU(5) through Scalar DM}

Despite the above predictions on neutrino masses and mixings, coupling unification, and proton lifetime, the SU(5) model with Higgs representations still has the vacuum instability problem. This problem in the SM arises as the standard Higgs potential solely controlled by the standard Higgs field becomes unstable for large values of the field at scales $\mu \geq$ $M_{\text {Inst. }}=5 \times 10^{9} \mathrm{GeV}$. As there is no other field so far in the extended SU(5) model for $\mu<M_{\Delta}\left(=10^{12}-10^{15} \mathrm{GeV}\right)$ to couple through its Higgs portal, the instability problem turns out to be similar to SM. As we have embedded the scalar singlet DM candidate in SU(5) we now investigate the possibility of resolving the vacuum instability through Higgs partial interaction $[88,89,149]$.

6.1. RG Equations and Parameters for Higgs Potential. As noted above the standard model Higgs potential

$$
V_{S M}=-\mu_{\phi}^{2} \phi^{\dagger} \phi+\lambda_{\phi}\left(\phi^{\dagger} \phi\right)^{2}
$$

develops instability as the Higgs quartic coupling $\lambda_{\phi}$ runs negative at an energy scale $10^{9}-10^{10} \mathrm{GeV}$ by the renormalization group running. Apart from other interesting suggestions $[88,89]$ an alternative popular solution to the vacuum instability problem is to extend the SM by a gauge singlet real scalar $(\xi)$ which gives positive contribution to the Higgs quartic coupling and prevents it from becoming negative $[88,162-164]$. It is worth mentioning that this scalar singlet can act as potential dark matter candidate termed as weakly interacting massive particle (WIMP) with an extra discrete symmetry $Z_{2}: \xi \longrightarrow-\xi$ imposed on it. The scalar singlet is odd under $Z_{2}$ symmetry while all other scalars being even and SM fermions being odd under this symmetry. Hence it can not couple to SM particle and become stable. This also matches the discrete symmetry properties of $\mathrm{SU}(5)$ representations discussed above. Thus it can serve as a suitable WIMP dark matter particle which is also identified as the SU(5) singlet scalar. The unbroken discrete symmetry of the singlet scalar upto the Planck scale has two important consequences: (i) the $\xi \mathrm{VEV}$ is forbidden and (ii) the modified SM potential develops VEV and minima only due to the SM Higgs. The scalar $\kappa(3,0,8)$ has no coupling with $\phi$. Even if $\Delta_{L}$ and some of its associates have coupling with $\phi$, because of their heavy mass, $M_{\Delta} \gg M_{W}$, they are treated to have decoupled from the Lagrangian at energy scales below $\mu \sim$ $M_{\Delta}$.

$\underline{\mu<M_{\Delta}}$

The potential of the model is modified in presence of the scalar singlet and a new term arises due to interaction of SM doublet $(\phi)$ with scalar singlet $(\xi)$ and self-interaction of $\xi$

$$
V(\xi, \phi)=V_{\mathrm{SM}}+\frac{\lambda_{\phi \xi}}{2} \phi^{\dagger} \phi \xi^{2}+\frac{\mu_{\xi}^{2}}{2} \xi^{2}+\frac{\lambda_{\xi}}{24} \xi^{4}
$$

where $\lambda_{\xi}$ is dark matter self-coupling, $\lambda_{\phi \xi}$ is standard Higgs and extra Higgs scalar interaction coupling or Higgs portal 
coupling and $\mu_{\xi}$ is quadratic coupling of extra Higgs scalar. From electroweak scale, up to $\mu=10^{12} \mathrm{GeV}$, the effective potential is $V^{\prime}(\xi, \phi)=V_{S M}+V(\xi, \phi)$.

$\underline{\mu>M_{\Delta}}$

The introduction of the scalar triplet $\Delta_{L}$ of mass $M_{\Delta} \sim$ $10^{12} \mathrm{GeV}$ changes the Higgs potential further by additional terms $V\left(\phi, \Delta_{L}\right)$ (arising out of interaction of SM doublet with scalar triplet and self-interaction of scalar triplet) and $V\left(\xi, \Delta_{L}\right)$ (arising out of interaction of scalar singlet DM and scalar triplet)

$$
V\left(\xi, \phi, \Delta_{L}\right)=V(\xi, \phi)+V\left(\phi, \Delta_{L}\right)+V\left(\xi, \Delta_{L}\right)
$$

where

$$
\begin{aligned}
V\left(\phi, \Delta_{L}\right)= & M_{\Delta}^{2} \operatorname{Tr}\left(\Delta_{L}^{\dagger} \Delta_{L}\right)+\frac{\lambda_{1}}{2}\left[\operatorname{Tr}\left(\Delta_{L}^{\dagger} \Delta_{L}\right)\right]^{2} \\
& +\frac{\lambda_{2}}{2}\left(\left[\operatorname{Tr}\left(\Delta_{L}^{\dagger} \Delta_{L}\right)\right]^{2}-\operatorname{Tr}\left[\left(\Delta_{L}^{\dagger} \Delta_{L}\right)^{2}\right]\right) \\
& +\lambda_{4}\left(\phi^{\dagger} \phi\right) \operatorname{Tr}\left(\Delta_{L}^{\dagger} \Delta_{L}\right) \\
& +\lambda_{5} \phi^{\dagger}\left[\Delta_{L}^{\dagger}, \Delta_{L}\right] \phi \\
& +\left(\mu_{\Delta} \widetilde{\phi}^{\dagger}\left(\frac{\vec{\tau} \cdot \overrightarrow{\Delta_{L}}}{\sqrt{2}}\right)^{\dagger} \phi+\text { h.c. }\right) \\
V\left(\xi, \Delta_{L}\right)= & \lambda_{\xi \Delta_{L}}\left(\xi^{\dagger} \xi\right)\left(\Delta_{L}^{\dagger} \Delta_{L}\right)
\end{aligned}
$$

Sufficiently below the mass scale $\mu=M_{\Delta}=10^{12} \mathrm{GeV}$, our model has two scalars: the first one is the SM Higgs $(\phi)$ given by $\phi=(1 / \sqrt{2})\left(\phi^{+}, v+h+i \phi^{0}\right)^{T}$ and the second one is extra scalar singlet $(\xi)$ added to the SM. The mass of the extra singlet is given by

$$
M_{D M}^{2}=\mu_{\xi}^{2}+\frac{\lambda_{\phi \xi}}{2} v^{2}
$$

We use the standard Higgs mass $m_{h}=125 \mathrm{GeV}$.

Direct detection experiments $[18,19]$ impose constraints on the Higgs portal coupling $\left(\lambda_{\phi \xi}\right)$ and dark matter mass [164, 165] derived from observed DM relic density

$$
M_{D M} \sim 3300 \times \lambda_{\phi \xi}
$$

or

$$
\lambda_{\phi \xi} \sim 0.0003 \times M_{D M}
$$

for $M_{D M} \gg m_{\text {top }}$. To be consistent with (41) we use $M_{D M}=$ $m_{\xi} \sim 1 \mathrm{TeV}$ throughout this work. Similar analysis can be carried out for all values of DM mass $>500 \mathrm{GeV}$.

These constraint on $\lambda_{\phi \xi}$ given in (48) can be also considerably relaxed if there is more than one WIMP DM candidate of the same or different species including fermions [110, 166].

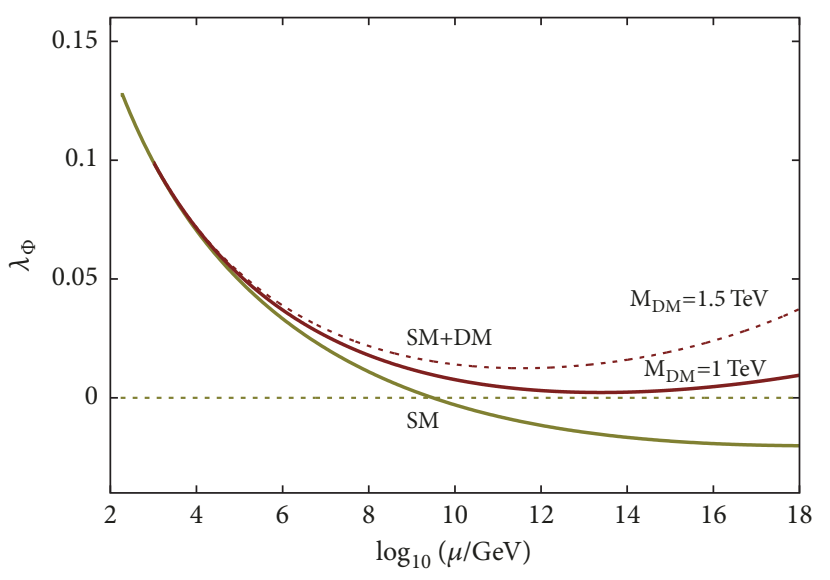

FIGURE 8: Running of Higgs quartic coupling.

6.2. RG Evolution of Quartic Coupling. Like other couplings of every non-Abelian gauge theory, it is well known that the SM Higgs potential is modified by quantum corrections determined by perturbative renormalization group equations (RGEs) for its running couplings $\eta(\mu)$

$$
\frac{d \eta}{d t}=\sum_{j} \frac{\eta^{(j)}}{\left(16 \pi^{2}\right)^{j}}
$$

where $t=\log \mu, \mu$ is renormalization scale, $\eta(\mu)=$ different couplings (quartic or gauge, or others) at scale $\mu$, and $j=$ $j^{\text {th }}$ loop order. The one-loop RG-coefficients of different couplings are presented in the Appendix. For the stability of the Higgs potential (see (43)), the value of self-coupling including corrections must remain positive throughout the course of its evolution up to the Planck scale.

The running of Higgs quartic coupling $\lambda_{\phi}(\mu)$ with energy scale $\mu$ is shown in Figure 8 .

In the figure, at first, we have neglected possible threshold effects due to Higgs triplet at $\mu=M_{\Delta}$ being determined as one of the solutions to neutrino oscillation data. Negligible $\Delta_{L^{-}}$ threshold effect can also result for $\mu_{\Delta} \ll M_{\Delta}$. We have used the initial values of different coupling constants at top-quark mass scale $\left(\mu=m_{\text {top }}\right)$ as given in Table 6 and subsequently evolved them from $m_{t}$ to Planck scale with the help of RGEs.

From Figure 8, it is clear that the desired quartic coupling remains stable up to the Planck scale for $\lambda_{\phi \xi}=0.36$ and $M_{D M}=1 \mathrm{TeV}$.

6.3. Higgs Triplet Threshold Effect. Threshold effect due to heavier Higgs masses which couple to $\phi$ through their portals has been discussed in general $[88,89]$ and in specific cases $[167,168]$. In our case the Higgs triplet mass used to fit the neutrino oscillation data is $M_{\Delta} \sim 10^{12} \mathrm{GeV}$ and its induced $\mathrm{VEV}$ is $\mathcal{O}(1-10) \mathrm{eV}$. In such a case the threshold effect caused by the triplet VEV correction term is [88]

$$
\Delta \lambda_{\phi}=\lambda_{\phi \Delta} \frac{v_{L}^{2}}{M_{\Delta}^{2}} \sim 10^{-36}
$$


TABLE 6: Initial values of coupling constants at top quark mass.

\begin{tabular}{lccccccc}
\hline Coupling constants & $\lambda_{\phi}\left(m_{t}\right)$ & $\lambda_{\xi}\left(m_{t}\right)$ & $\lambda_{\phi \xi}\left(m_{t}\right)$ & $g_{1 Y}\left(m_{t}\right)$ & $g_{2 L}\left(m_{t}\right)$ & $g_{3 C}\left(m_{t}\right)$ & $y_{t}\left(m_{t}\right)$ \\
\hline Initial values & 0.1296 & 0.1 & 0.36 & 0.35 & 0.64 & 1.16 & 0.94 \\
\hline
\end{tabular}

The remaining threshold effect could be due the self-energy correction or the trilinear term $\mu_{\Delta} \Delta_{L} \phi \phi+$ h.c in the Higgs potential giving rise to threshold correction to quartic coupling

$$
\Delta \lambda_{\phi} \equiv \lambda_{\mathrm{TH}}=\frac{\mu_{\Delta}^{2}}{M_{\Delta}^{2}}
$$

Denoting the effective Higgs quartic coupling by $\lambda^{\prime}(\mu)$ for $\mu \geq M_{\Delta}$ this is related to the quartic coupling $\lambda_{\phi}(\mu)$ at $\mu=M_{\Delta}$ [88]

$$
\lambda_{\phi}\left(M_{\Delta}\right)=\lambda^{\prime}\left(M_{\Delta}\right)-\lambda_{\mathrm{TH}} .
$$

This correction comes into play when the running mass scale is $\mu \sim M_{\Delta}$ and larger.

We point out that the same values of Majorana Yukawa coupling elements of $Y$ derived in Section 2 are valid up to a scale factor for a wide range of values of trilinear coupling mass parameter $\mu_{\Delta}<M_{\Delta}^{0}$ for which this threshold effect is well within the perturbative regime. We note from (8) that the mass formula gives the scaling relation

$$
Y=Y^{0} \frac{v_{L}^{0}}{v_{L}}=Y^{0} \frac{\mu_{\Delta}^{0}}{\mu_{\Delta}}
$$

where we have used the zero superscript for values at $\mu_{\Delta}^{0}=$ $M_{\Delta}=10^{12} \mathrm{GeV}$. Thus, for the values of neutrino mass and mixing given by the oscillation data, a new set of elements of $Y$ are derived for any $\mu_{\Delta}<\mu_{\Delta}^{0}$ by multiplying all the vales given in Tables 2 and 3 by the same scale factor $\mu_{\Delta}^{0} / \mu_{\Delta}$.

In Figure 9 we have presented evolution of Higgs quartic couplings below and above $\mu=M_{\Delta}$ for $\lambda_{\mathrm{TH}}=0.1$. Using the notations of the Appendix, we have used the initial values of different coupling constants at scalar triplet mass scale $\left(M_{\Delta}\right)$ as $\lambda_{1}=\lambda_{2}=\lambda_{4}=0.1$ and $\lambda_{5}=0.1$.

For all the three curves given in Figure 9 the scalar DM mass has been fixed at $M_{\mathrm{DM}}=1 \mathrm{TeV}$ consistent with LUX:2016 data. The curve labeled as $\mathrm{SM}+\mathrm{DM}+\Delta$ includes threshold effect $\lambda_{\mathrm{TH}}=0.1$ at $\mu=M_{\Delta}=10^{12} \mathrm{GeV}$ corresponding to $\mu_{\Delta} \sim(1 / 3) M_{\Delta}$. We have checked that even after including the heavy scalar threshold effect the quartic coupling remains perturbatively positive upto the Planck scale for $\mu_{\Delta} / M_{\Delta} \simeq 0.5$. (Denoting $\Phi_{H}=24_{H}$, above the mass scale $\mu>M_{\mathrm{GUT}}$ we impose the well known discrete symmetry $\Phi_{H} \longrightarrow-\Phi_{H}$ which is usually assumed in the minimal SU(5) model. Without loss of generality we further assume the Higgs portal coupling $\lambda_{5_{H}, 75_{H}}$ to be negligible.)

Thus, the issue of vacuum stability of SM Higgs potential is resolved through the embedding of $\xi$ as a WIMP dark matter candidate in $\mathrm{SU}(5)$ even after including the heavy Higgs triplet threshold effect which could be verified by direct search experiments and LHC.

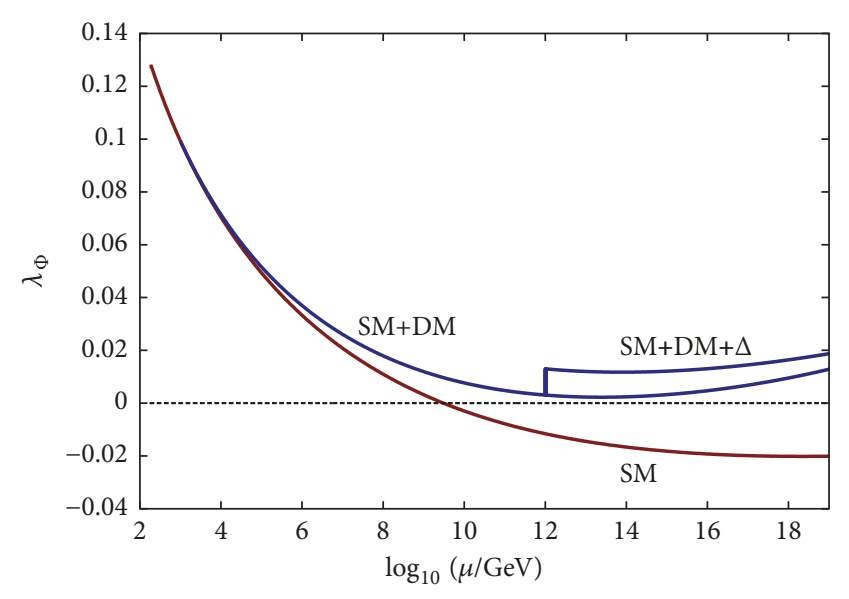

FIGURE 9: Running of standard Higgs quartic coupling including heavy triplet scalar threshold effect at $\mu=M_{\Delta}=10^{12} \mathrm{GeV}$ derived from fits to neutrino oscillation data. The curves labeled as SM, $\mathrm{SM}+\mathrm{DM}$, and $\mathrm{SM}+\mathrm{DM}+\Delta$ denote contributions due to $\mathrm{SM}$ alone, SM plus DM, and SM plus DM plus Higgs triplet threshold effect, respectively, as described in the text. The scalar DM mass has been fixed at $M_{\mathrm{DM}}=1.0 \mathrm{TeV}$ consistent with LUX:2016 experimental data.

\section{Summary and Conclusion}

In this work we have attempted to resolve four limitations of the minimal $\mathrm{SU}(5)$ model by extending its scalar sector beyond $5_{H}$ and $24_{H}$. Added presence of $15_{H}$ and $75_{H}$ is noted to account for precision coupling unification with experimentally verifiable proton lifetime for $p \longrightarrow e^{+} \pi^{0}$, and type-II seesaw ansatz for neutrino masses. The left-handed triplet Higgs mass in this model is bounded from below $\left(M_{\Delta}=M_{15_{H}}\right) \geq\left(M_{\kappa}=10^{9.23}\right) \mathrm{GeV}$. Proton lifetime is predicted by taking into account sources of theoretical uncertainties due to GUT threshold effects and those due to electroweak precision parameters. Type-II seesaw scale effect on proton lifetime prediction is also discussed. The limitation due to vacuum stability of the Higgs potential in $\mathrm{SU}(5)$ is resolved by the inclusion of a scalar singlet near the $\mathrm{TeV}$ scale that acts as a WIMP dark matter candidate. All the fermions and this scalar are assigned to be odd under a dark matter stabilising $Z_{2}$ discrete symmetry whereas the SM Higgs is even. The scalar dark matter mass is consistent with current experimental LUX-2016 bound on direct search experiments. Renormalization group evolution of SM Higgs quartic coupling modified by Higgs portal coupling of this scalar DM completely alleviates the vacuum instability problem. We emphasize that no nonstandard Higgs field, except the scalar DM singlet, is present in this model below the $\kappa$ mass $M_{\kappa}=10^{9.23} \mathrm{GeV}$. 
We thus conclude that such SM limitations as neutrino mass, coupling unification, proton lifetime, WIMP dark matter, and vacuum stability can be effectively resolved by extending the scalar sector of $\mathrm{SU}(5)$ to include $5_{H}, 24_{H}, 75_{H}, 15_{H}$, and $1_{H}$. At present we need no extension on the established fermion structure of the SM and SU(5) or their minimal gauge structure. The remaining limitations on baryon asymmetry generation and/or the possibility of decaying dark matter projected to manifest as $\mathrm{PeV}$ energy IceCube neutrinos will be addressed elsewhere $[169,170]$.

\section{Appendix}

\section{Renormalization Group Equations for Higgs Scalar Couplings}

The RGEs for scalar quartic couplings $[60-62,168]$ in our model at one loop level are given by

$$
16 \pi^{2} \frac{d C}{d t}=\beta_{C} \quad\left(C=\lambda_{\phi}, \lambda_{\phi \xi}, \lambda_{\xi}, \lambda_{1}, \lambda_{2}, \lambda_{4}, \lambda_{5}\right)
$$

where

$$
\begin{aligned}
& \beta_{\lambda_{\phi}} \\
& =24 \lambda_{\phi}^{2}+12 \lambda_{\phi} y_{t}^{2}-6 y_{t}^{4}-3 \lambda_{\phi}\left(g_{1 Y}^{2}+3 g_{2 L}^{2}\right) \\
& \quad+\frac{3}{8}\left[2 g_{2 L}^{4}+\left(g_{1 Y}^{2}+g_{2 L}^{2}\right)^{2}\right]+\frac{\lambda_{\phi \xi}^{2}}{2} \\
& \beta_{\lambda_{\phi \xi}} \\
& =\left\{4 \lambda_{\phi \xi}+12 \lambda_{\phi}+6 y_{t}^{2}-\frac{3}{2}\left(g_{1 Y}^{2}+3 g_{2 L}^{2}\right)+\lambda_{\xi}\right\} \lambda_{\phi \xi} \\
& \beta_{\lambda_{\xi}}=3 \lambda_{\xi}^{2}+12 \lambda_{\phi \xi}^{2}
\end{aligned}
$$

For Standard model RG running in the energy scale $\mu<$ $M_{D M}$, the term $\lambda_{\phi \xi} / 2$ in $\beta_{\lambda_{\phi}}$ in (A.3) is to be ignored. The RGEs for SM gauge couplings and top-quark Yukawa coupling at two-loop level are given by

$$
\begin{aligned}
\frac{d y_{t}}{d t} & =\frac{1}{16 \pi^{2}}\left(\frac{9}{2} y_{t}^{2}-\frac{17}{12} g_{1 Y}^{2}-\frac{9}{4} g_{2 L}^{2}-8 g_{3 C}^{2}\right) y_{t} \\
& +\frac{1}{\left(16 \pi^{2}\right)^{2}}\left[-\frac{23}{4} g_{2 L}^{4}-\frac{3}{4} g_{2 L}^{2} g_{1 Y}^{2}+\frac{1187}{216} g_{1 Y}^{4}\right. \\
& +9 g_{2 L}^{2} g_{3 C}^{2}+\frac{19}{9} g_{3 C}^{2} g_{1 Y}^{2}-108 g_{3 C}^{4} \\
& +\left(\frac{225}{16} g_{2 L}^{2}+\frac{131}{16} g_{1 Y}^{2}+36 g_{3 C}^{2}\right) y_{t}^{2} \\
& \left.+6\left(-2 y_{t}^{4}-2 y_{t}^{2} \lambda_{\phi}+\lambda_{\phi}^{2}\right)\right]
\end{aligned}
$$

$$
\begin{aligned}
& \frac{d g_{1 Y}}{d t}=\frac{1}{16 \pi^{2}}\left(\frac{41}{6} g_{1 Y}^{3}\right)+\frac{1}{\left(16 \pi^{2}\right)^{2}}\left(\frac{199}{18} g_{1 Y}^{2}\right. \\
& \left.\quad+\frac{9}{2} g_{2 L}^{2}+\frac{44}{3} g_{3 C}^{2}-\frac{17}{6} y_{t}^{2}\right) g_{1 Y}^{3} \\
& \frac{d g_{2 L}}{d t}=\frac{1}{16 \pi^{2}}\left(-\frac{19}{6} g_{2 L}^{3}\right)+\frac{1}{\left(16 \pi^{2}\right)^{2}}\left(\frac{3}{2} g_{1 Y}^{2}\right. \\
& \left.\quad+\frac{35}{6} g_{2 L}^{2}+12 g_{3 C}^{2}-\frac{3}{2} y_{t}^{2}\right) g_{2 L}^{3} \\
& \frac{d g_{3 C}}{d t}=\frac{1}{16 \pi^{2}}\left(-7 g_{3 C}^{3}\right)+\frac{1}{\left(16 \pi^{2}\right)^{2}}\left(\frac{11}{6} g_{1 Y}^{2}+\frac{9}{2} g_{2 L}^{2}\right. \\
& \left.\quad-26 g_{3 C}^{2}-2 y_{t}^{2}\right) g_{3 C}^{3}
\end{aligned}
$$

After $\mu=10^{12} \mathrm{GeV}$ the scalar triplet $\Delta_{L}$ is introduced and we use the modified RG equations of $\lambda_{\phi}$ and other couplings relevant for this scalar triplet.

$$
\begin{aligned}
\beta_{\lambda_{\phi}} & =\lambda_{\phi}\left[12 \lambda_{\phi}-\left(\frac{9}{5} g_{1 Y}^{2}+9 g_{2 L}^{2}\right)+12 y_{t}^{2}\right] \\
& +\frac{9}{4}\left(\frac{3}{25} g_{1 Y}^{4}+\frac{2}{5} g_{1 Y}^{2} g_{2 L}^{2}+g_{2 L}^{4}\right)+6 \lambda_{4}^{2}+4 \lambda_{5}^{2} \\
& -12 y_{t}^{4}, \\
\beta_{\lambda_{1}} & =\lambda_{1}\left[14 \lambda_{1}+4 \lambda_{2}-\left(\frac{36}{5} g_{1 Y}^{2}+24 g_{2 L}^{2}\right)\right. \\
& +4 \operatorname{tr}[T]]+\frac{108}{25} g_{1 Y}^{4}+\frac{72}{5} g_{1 Y}^{2} g_{2 L}^{2}+18 g_{2 L}^{4}+2 \lambda_{2}^{2} \\
& +4 \lambda_{4}^{2}+4 \lambda_{5}^{2}-8 \operatorname{tr}\left[T^{2}\right], \\
\beta_{\lambda_{2}} & =\lambda_{2}\left[12 \lambda_{1}+3 \lambda_{2}-\left(\frac{36}{5} g_{1 Y}^{2}+24 g_{2 L}^{2}\right)\right. \\
& +4 \operatorname{tr}[T]]-\frac{144}{5} g_{1 Y}^{2} g_{2 L}^{2}+12 g_{2 L}^{4}-8 \lambda_{5}^{2} \\
& \left.+6 y_{t}^{2}+2 \operatorname{tr}[T]\right]-\frac{18}{5} g_{1 Y}^{2} g_{2 L}^{2}+4 \operatorname{tr}\left[T^{2}\right] \\
& +8 \operatorname{tr}\left[T^{2}\right], \\
\beta_{\lambda_{5}} & =\lambda_{5}\left[2 \lambda+2 \lambda_{1}-2 \lambda_{5}^{2}-4 \operatorname{tr}\left[T^{2}\right],\right. \\
& =\left(\frac { 9 } { 2 } g _ { 1 Y } ^ { 2 } \left[6 \lambda_{\phi}+8 \lambda_{1}+2 \lambda_{2}+4 \lambda_{4}\right.\right. \\
& \left.\left.+33 g_{2 L}^{2}\right)+6 y_{t}^{2}+2 \operatorname{tr}[T]\right]+\frac{27}{25} g_{1 Y}^{4} \\
& \left.+\frac{9}{2} g_{1 Y}^{2}+\frac{33}{2 L}\right) \\
& \\
&
\end{aligned}
$$


where $T$ is defined as $T=Y^{\dagger} Y$ and its beta function is expressed through the relation

$$
\beta_{T}=T\left[6 T-3\left(\frac{3}{5} g_{1 Y}^{2}+3 g_{2 L}^{2}\right)+2 \operatorname{tr}[T]\right] .
$$

\section{Data Availability}

The data used to support the findings of this study are available from the corresponding author upon request.

\section{Conflicts of Interest}

The authors declare that they have no conflicts of interest.

\section{Acknowledgments}

M. K. Parida acknowledges financial support under the Project ddd SB/S2/HEP-011/2013 from the Department of Science and Technology, Government of India. Financial support from Siksha 'O' Anusandhan (SOA), Deemed to be University, to Mainak Chakraborty for a Postdoctoral fellowship and to Biswonath Sahoo for a $\mathrm{Ph}$. D. research fellowship is acknowledged.

\section{References}

[1] P. F. de Salas, D. V. Forero, C. A. Ternes, M. Tortola, and J. W. Valle, "Status of neutrino oscillations 2018: $3 \sigma$ hint for normal mass ordering and improved CP sensitivity", Physics Letters. $B$. Particle Physics, Nuclear Physics and Cosmology, vol. 782, pp. 633-640, 2018.

[2] T. Schwetz, M. Tórtola, and J. W. Valle, "Global neutrino data and recent reactor fluxes: the status of three-flavour oscillation parameters," New Journal of Physics, vol. 13, no. 6, p. 063004, 2011.

[3] D. Forero, M. Tortola, and J. Valle, "Neutrino oscillations refitted," Physical Review D: Particles, Fields, Gravitation and Cosmology, vol. 90, no. 9, Article ID 093006, 10 pages, 2014.

[4] G. L. Fogli, E. Lisi, A. Marrone, D. Montanino, A. Palazzo, and A. M. Rotunno, "Global analysis of neutrino masses, mixings and phases: entering the era of leptonic CP violation searches," Physical Review D: Particles, Fields, Gravitation and Cosmology, vol. 86, Article ID 013012, 2012.

[5] J. Bergström, M. C. Gonzalez-Garcia, M. Maltoni, and T. Schwetz, "Bayesian global analysis of neutrino oscillation data," Journal of High Energy Physics, vol. 2015, no. 9, 2015.

[6] F. Zwicky, "Die rotverschiebung von extragalaktischen nebeln," Helvetica Physica Acta, vol. 6, pp. 110-127, 1933.

[7] F. Zwicky, "Republication of: The redshift of extragalactic nebulae," General Relativity and Gravitation, vol. 41, pp. 207224, 2009.

[8] WMAP Collaboration, D. N. Spergel et al., "Wilkinson Microwave Anisotropy Probe(WMAP) three year results: implications for cosmology," The Astrophysical Journal Supplement Series, vol. 170, Article ID 0603449, p. 377, 2007.

[9] J. Einasto, “"Dark Matter”, Astronomy and Astrophysics 2010," in Encyclopedia of Life Support Systems (EOLSS), O. Engvold, R. Stabell, B. Czerny, and J. Lattanzio, Eds., Eolss Publishers, Oxford,UK, 2010, Developed under the Auspices of the UNESCO.
[10] G. R. Blumenthal, S. M. Faber, J. R. Primack, and M. J. Rees, "Formation of galaxies and large-scale structure with cold dark matter," Nature, vol. 311, no. 5986, pp. 517-525, 1984.

[11] J. Angle et al., "A search for light dark matter in XENON10 data," Physical Review Letters, vol. 107, Article ID 051301, 2011.

[12] J. Angle et al., "Erratum: Search for light dark matter in XENON10 data," Physical Review Letters, vol. 110, 2013.

[13] L. E. Strigari, "Galactic searches for dark matter," Physics Reports, vol. 531, no. 1, pp. 1-88, 2013.

[14] V. C. Rubin and W. K. Ford, "Rotation of the andromeda nebula from a spectroscopic survey of emission regions," The Astrophysical Journal, vol. 159, p. 379, 1970.

[15] D. Clowe, M. Bradač, A. H. Gonzalez et al., "A direct empirical proof of the existence of dark matter," The Astrophysical Journal, vol. 648, no. 2, pp. L109-L113, 2006.

[16] E. Aprile et al., "Dark Matter Results from 225 Live Days of XENON100 Data," Physical Review Letters, vol. 109, Article ID 181301, 2012.

[17] D. S. Akerib et al., "First results from the LUX dark matter experiment at the Sanford Underground Research Facility," Physical Review Letters, vol. 112, Article ID 091303, 2014.

[18] D. S. Akerib et al., "Results from a Search for Dark Matter in the Complete LUX Exposure," Physical Review Letters B, vol. 118, no. 2, Article ID 021303, 2017.

[19] M. Ajello et al., "Fermi-LAT Observations of High Energy $\gamma$ ray Emission Toward the Galactic Center," The Astrophysical Journal, vol. 819, no. 1, p. 44, 2016, arXiv:1511.02938.

[20] M. G. Aartsen, "Evidence for high-energy extraterrestrial neutrinos at the IceCube detector," Science, vol. 342, no. 6161, Article ID 1242856, 2013.

[21] M. G. Artsen et al., "Observation of High-Energy Astrophysical Neutrinos in Three Years of IceCube Data," Physical Review Letters, vol. 113, Article ID 101101, 2014.

[22] M. G. Artsen et al., "Lowering IceCube's Energy Threshold for Point Source Searches in the Southern Sky," The Astrophysical Journal, vol. 824, no. 2, 2016.

[23] IceCube, ANTARES Collaboration, S. Adrian-Martinez et al., "The First Combined Search for Neutrino-Point Sources in the Southern Hemisphere," The Astrophysical Journal, vol. 823, no. 1, p. 65, 2016.

[24] CMS Collaboration, A. M. Sirunyan et al., "Search for dark matter produced in association with heavy-flavor quarks in protonproton collisions at sqrt(s)=13 TeV," High Energy Physics, 2017, CMS-EXO-16-005, CERN-EP-2017-087.

[25] A. M. Sirunyan et al., "Search for associated production of dark matter with a Higgs boson decaying to bb or $\gamma \gamma$ at $s=13 \mathrm{TeV}$," in JHEP, vol. 1710, 180 edition, 2017, arXiv:1703.05236.

[26] D. N. Spergel et al., "First year Wilkinson Microwave Anisotropy Probe (WMAP) Observations: Determination of Cosmological Parameters," The Astrophysical Journal Supplement Series, vol. 148, p. 175, 2003.

[27] WMAP Collaboration, E. Komatsu et al., "Seven-Year Wilkinson Microwave Anisotropy Probe (WMAP) Observations: Cosmological Interpretation," The Astrophysical Journal Supplement Series, vol. 192, p. 18, 2011, arXiv:1001.4538.

[28] WMAP Collaboration, G. Hindshaw et al., "Five-Year Wilkinson Microwave Anisotropy Probe (WMAP) Observations: Data Processing, Sky Maps and Basic Results," The Astrophysical Journal Supplement Series, vol. 180, p. 225, 2009, arXiv:0803.0732.

[29] Planck Collaboration, P. A. Ade et al., "Planck 2015 results. XIII. Cosmological parameter," Astron Astrophys, vol. 59, p. pp, 2015, arXiv:1502.01589. 
[30] J. C. Pati and A. Salam, "Lepton number as the fourth 'color," Physical Review D: Particles, Fields, Gravitation and Cosmology, vol. 10, no. 1, pp. 275-289, 1974.

[31] J. C. Pati and A. Salam, "Erratum: Lepton number as the fourth 'color"' Physical Review D: Particles, Fields, Gravitation and Cosmology, vol. 11, pp. 275-289, 1975.

[32] H. Georgi and S. L. Glashow, "Unity of all elementary-particle forces," Physical Review Letters, vol. 32, no. 8, pp. 438-441, 1974.

[33] H. Georgi, “The State of the Art-Gauge Theories," in Particles and Fields, Williamsburg, Virginia (1974), AIP Conf. Proc. 23 (1975) 575.

[34] H. Fritzsch and P. Minkowski, "Unified interactions of leptons and hadrons," Annals of Physics, vol. 93, no. 1-2, pp. 193-266, 1975.

[35] P. Langacker, "Grand unified theories and proton decay," Physics Reports, vol. 72, no. 4, pp. 185-385, 1981.

[36] R. Slansky, "Group theory for unified model building," Physics Reports, vol. 79, no. 1, pp. 1-128, 1981.

[37] P. Nath and P. Fileviez Perez, "Proton stability in grand unified theories, in strings and in branes," Physics Reports, vol. 441, no. 5-6, pp. 191-317, 2007.

[38] G. Senjanovic, "Neutrino mass: from LHC to grand unification," La Rivista del Nuovo Cimento, vol. 34, pp. 1-68, 2011.

[39] G. Senjanovic and V. Tello, "Origin of Neutrino Mass," in Proceedings of the 18th International Conference From the Planck Scale to the Electroweak Scale, p. 141, Ioannina, Greece, May 2015.

[40] G. Altarelli, "Neutrinos Today: An Introduction," in Proceedings of the in Proceedings: 49th Recontres de Moriond on Electroweak Interactons and Unified Theories, Thuile, Italy, 2014.

[41] A. Y. Smirnov, "Theories of Neutrino Masses and Mixings," Il Nuovo Cimento C, vol. 37, no. 3, 2014.

[42] R. N. Mohapatra, "From old symmetries to new symmetries: Quarks, leptons and B - L," 50 Years of Quarks, pp. 245-263, 2015.

[43] R. N. Mohapatra, "Neutrino mass as a signal of TeV scale physics," Nuclear Physics B, vol. 908, pp. 423-435, 2016.

[44] O. G. Miranda and J. W. Valle, "Neutrino oscillations and the seesaw origin of neutrino mass," Nuclear Physics B, vol. 908, pp. 436-455, 2016.

[45] M. K. Parida and B. P. Nayak, "Singlet Fermion Assisted Dominant Seesaw with Lepton Flavor and Number Violations and Leptogenesis," Advances in High Energy Physics, vol. 2017, Article ID 4023493, 24 pages, 2017.

[46] P. Minkowski, " $\mu \longrightarrow \mathrm{e} \gamma$ at a rate of one out of $10^{9}$ muon decays?" Physics Letters B, vol. 67, pp. 421-428, 1977.

[47] M. Gell-Mann, P. Ramond, and R. Slansky, Supergravity, P. van Nieuwenhuizen and D. Freedman, Eds., North-Holland, 1979.

[48] S.L. Glashow, in Quarks and Leptons, Cargèse, eds. M. Lévy et al., Plenum, 1980, New-York, p. 707.

[49] T. Yanagida, in Proceedings of the Workshop on the Unified Theory and the Baryon Number in the Universe, O. Sawada and A. Sugamoto, Eds., p. 95, Tsukuba, 1979, KEK Report No. 79-18.

[50] R. N. Mohapatra and G. Senjanovic, "Neutrino mass and spontaneous parity nonconservation," Physical Review Letters, vol. 44, p. 912, 1980.

[51] J. Schechter and J. W. F. Valle, "Neutrino masses in $\mathrm{SU}(2) \otimes \mathrm{U}(1)$ theories," Physical Review D: Particles, Fields, Gravitation and Cosmology, vol. 22, p. 2227, 1980.

[52] J. Schechter and J. W. F. Valle, "Neutrino decay and spontaneous violation of lepton number," Physical Review D: Particles, Fields, Gravitation and Cosmology, vol. 25, p. 774, 1982.
[53] T. Cheng and L.-F. Li, "Neutrino masses, mixings, and oscillations in $\mathrm{SU}(2) \times \mathrm{U}(1)$ models of electroweak interactions," Physical Review D: Particles, Fields, Gravitation and Cosmology, vol. 22, no. 11, pp. 2860-2868, 1980.

[54] M. Magg, C. Wetterich, and B. Phys. Lett, "Neutrino mass problem and gauge hierarchy," Physics Letters B, vol. 94, pp. 6164, 1980.

[55] G. Lazarides, Q. Shafi, and C. Wetterich, "Proton lifetime and fermion masses in an SO(10) model," Nuclear Physics Section B, vol. 181, no. 2, pp. 287-300, 1981.

[56] R. N. Mohapatra and G. Senjanović, "Neutrino masses and mixings in gauge models with spontaneous parity violation," Physical Review D: Particles, Fields, Gravitation and Cosmology, vol. 23, article 165, 1981.

[57] E. Ma and U. Sarkar, "Neutrino masses and leptogenesis with heavy higgs triplets," Physical Review Letters, vol. 80, no. 26, article 5716, 1998.

[58] R. Foot, H. Lew, X. G. He, and G. C. Joshi, "See-saw neutrino masses induced by a triplet of leptons," Zeitschrift für Physik C: Particles and Fields, vol. 44, no. 3, pp. 441-444, 1989.

[59] E. Ma, "Pathways to naturally small neutrino masses," Physical Review Letters, vol. 81, p. 1171, 1998.

[60] B. Bajc and G. Senjanovic, "Seesaw at LHC," Journal of High Energy Physics. A SISSA Journal, vol. 0708, no. 014, 2007.

[61] A. Arhrib, B. Bajc, D. K. Ghosh et al., " Collider signatures for the heavy lepton triplet in the type " Physical Review D: Particles, Fields, Gravitation and Cosmology, vol. 82, no. 5, 2010.

[62] B. Bajc, M. Nemevsek, and G. Senjanovic, "Probing seesaw at LHC," Physical Review D, vol. 76, Article ID 055011, 2007.

[63] E. Akhmedova, M. Lindnerb, E. Schnapkab, and J. Vallec, "Leftright symmetry breaking in NJL approach," Physics Letters B, vol. 368, no. 4, pp. 270-280, 1996.

[64] M. Malinsky, J. Romao, and J. Valle, "Supersymmetric SO(10) seesaw mechanism with low $B-L$ scale," Physical Review Letters, vol. 95, Article ID 161801, 2005.

[65] R. N. Mohapatra and J. W. F. Valle, "Neutrino mass and baryon-number nonconservation in superstring models," Physical Review D: Particles, Fields, Gravitation and Cosmology, vol. 34, no. 5, pp. 1642-1645, 1986.

[66] R. N. Mohapatra, "Mechanism for understanding small neutrino mass in superstring theories," Physical Review Letters, vol. 56, no. 6, pp. 561-563, 1986.

[67] C. H. Albright, "Search for a solution of the superstring neutrino mass problem without a high intermediate energy scale," Physics Letters B, vol. 178, no. 2-3, pp. 219-225, 1986.

[68] E. Witten, "New issues in manifolds of SU(3) holonomy," Nuclear Physics B, vol. 268, no. 1, pp. 79-112, 1986.

[69] S. Nandi and U. Sarkar, "Solution to the neutrino-mass problem in superstring E6 theory," Physical Review Letters, vol. 56, no. 6, pp. 564-567, 1986.

[70] E. Ma, "Lepton-number nonconservation in E6 superstring models," Physics Letters B, vol. 191, no. 3, pp. 287-289, 1987.

[71] D. Wyler and L. Wolfenstein, "Massless neutrinos in left-hand symmetric models," Nuclear Physics B, vol. 218, no. 1, pp. 205214, 1983.

[72] J. Bernabeu, A. Santamaria, and J. Vidal, "Lepton flavour nonconservation at high energies in a superstring inspired standard model," Physics Letters B, vol. 187, pp. 303-308, 1987.

[73] J. W. F. Valle, "Gauge theories and physics of neutrino mass," Progress in Particle and Nuclear Physics, pp. 91-171, 1991. 
[74] M. K. Parida, R. L. Awasthi, and P. K. Sahu, "Proton decay and new contributions to $0 \nu 2 \beta$ decay in $\mathrm{SO}(10)$ with low-mass $Z^{\prime}$ boson, observable $n-\bar{n}$ oscillation, lepton flavor violation, and rare kaon decay," Journal of High Energy Physics, vol. 2015, article 45, 2015.

[75] R. L. Awasthi and M. K. Parida, "Inverse seesaw mechanism in nonsupersymmetric $\mathrm{SO}(10)$, proton lifetime, nonunitarity effects, and a low-mass $Z^{\prime}$ boson," Physical Review D: Particles, Fields, Gravitation and Cosmology, vol. 86, no. 9, Article ID 093004, 2012.

[76] M. Reig, D. Restrepo, J. W. F. Valle, and O. Zapata, Bound-state dark matter and Dirac neutrino mass, arXiv:1803.08528.

[77] S. Centelles Chuliá, R. Srivastava, and J. W. Valle, "Seesaw roadmap to neutrino mass and dark matter," Physics Letters. B. Particle Physics, Nuclear Physics and Cosmology, vol. 781, pp. 122-128, 2018.

[78] S. Weinberg, Living in the Universe, or Multi Universe, B. J. Carr, Ed., Cambridge University Press, 2007.

[79] S. M. Barr, "New approach to flavor symmetry and an extended naturalness principle," Physical Review D: Particles, Fields, Gravitation and Cosmology, vol. 82, no. 5, 2010.

[80] D. Chang, R. N. Mohapatra, and M. K. Parida, "Decoupling of parity- and $\mathrm{SU}(2) \mathrm{R}$-breaking scales: a new approach to left-right symmetric models," Physical Review Letters, vol. 52, no. 13, pp. 1072-1075, 1984.

[81] D. Chang, R. N. Mohapatra, and M. K. Parida, "New approach to left-right-symmetry breaking in unified gauge theories," Physical Review D: Particles, Fields, Gravitation and Cosmology, vol. 30 , no. 5, article 1052, 1984.

[82] D. Chang, R. N. Mohapatra, J. M. Gipson, R. E. Marshak, and M. K. Parida, "Experimental tests of new SO(10) grand unification," Physical Review D: Particles, Fields, Gravitation and Cosmology, vol. 31, no. 7, pp. 1718-1732, 1985.

[83] P. Langacker and N. Polonsky, "Uncertainties in coupling constant unification," Physical Review D: Particles, Fields, Gravitation and Cosmology, vol. 47, no. 9, pp. 4028-4045, 1993.

[84] E. Ma and D. Suematsu, "Fermion triplet dark matter and radiative neutrino mass," Modern Physics Letters A, vol. 24, no. 8, pp. 583-589, 2009.

[85] D. Meloni, T. Ohlsson, and S. Riad, "Renormalization Group Running of Fermion Observables in an Extended NonSupersymmetric SO(10) Model," in JHEP, vol. 3, p. 45, 2017, arXiv:1612.07973.

[86] C. Das and M. Parida, "New formulas and predictions for running fermion masses at higher scales in SM, 2HDM, and MSSM," The European Physical Journal C, vol. 20, no. 1, pp. 121137, 2001.

[87] M. K. Parida, P. K. Patra, and A. K. Mohanty, "Gravity-induced large grand-unification mass in SU(5) with higher-dimensional operators," Physical Review D: Particles, Fields, Gravitation and Cosmology, vol. 39, no. 1, pp. 316-322, 1989.

[88] J. Elias-Miró, J. R. Espinosa, G. F. Giudice, H. M. Lee, and A. Strumia, "Stabilization of the electroweak vacuum by a scalar threshold effect," Journal of High Energy Physics, vol. 2012, article 31, 2012.

[89] O. Lebedev, "On stability of the electroweak vacuum and the Higgs portal," The European Physical Journal C, vol. 72, no. 7, 2012.

[90] S. Chigusa, T. Moroi, and Y. Shoji, "Decay rate of electroweak vacuum in the standard model and beyond," Physical Review D: Particles, Fields, Gravitation and Cosmology, vol. 97, no. 11, 2018.
[91] E. Ma, "Efficacious additions to the Standard Model," Physics Letters B, vol. 625, no. 1-2, pp. 76-78, 2005.

[92] I. Doršner, S. Fajfer, A. Greljo, J. Kamenik, and N. Košnik, "Physics of leptoquarks in precision experiments and at particle colliders," Physics Reports, vol. 641, pp. 1-68, 2016.

[93] I. Doršner, “Scalar leptoquark in ," Physical Review D: Particles, Fields, Gravitation and Cosmology, vol. 86, no. 5, 2012.

[94] I. Doršner, S. Fajfer, and I. Mustać, “ Light vectorlike fermions in a minimal ", Physical Review D: Particles, Fields, Gravitation and Cosmology, vol. 89, no. 11, 2014.

[95] I. Doršner, S. Fajfer, and N. Košnik, "Leptoquark mechanism of neutrino masses within the grand unification framework," The European Physical Journal C, vol. 77, no. 6, 2017.

[96] I. Doršner, S. Fajfer, and N. Košnik, "Heavy and light scalar leptoquarks in proton decay," Physical Review D: Particles, Fields, Gravitation and Cosmology, vol. 86, Article ID 015013, 2012.

[97] I. Doršner and P. F. Pérez, "Upper bound on the mass of the type III seesaw triplet in an SU(5) model," Journal of High Energy Physics, vol. 2007, 2007.

[98] P. F. Pérez, "Supersymmetric adjoint SU(5)," Physical Review D: Particles, Fields, Gravitation and Cosmology, vol. 76, no. 7, 2007.

[99] W.-Y. Keung and G. Senjanović, "Majorana neutrinos and the production of the right-handed charged gauge boson," Physical Review Letters, vol. 50, no. 19, pp. 1427-1430, 1983.

[100] S. Weinberg, "Effective gauge theories," Physics Letters B, vol. 91, no. 1, pp. 51-55, 1980.

[101] L. J. Hall, "Grand unification of effective gauge theories," Nuclear Physics B, vol. 178, no. 1, pp. 75-124, 1981.

[102] B. A. Ovrut and H. J. Schnitzer, "Effective field theory in background field gauge," Physics Letters B, vol. 110, no. 2, pp. 139-142, 1982.

[103] M. K. Parida, "Heavy particle effects in grand unified theories with fine-structure constant matching," Physics Letters B, vol. 196, no. 2, pp. 163-169, 1987.

[104] M. K. Parida and P. K. Patra, " Useful theorem on vanishing threshold contribution to ", Physical Review Letters, vol. 66, no. 7, pp. 858-861, 1991.

[105] M. K. Parida and P. K. Patra, “Theorem on vanishing multiloop radiative corrections to ", Physical Review Letters, vol. 68, no. 6, pp. 754-756, 1992.

[106] M. K. Parida and C. C. Hazra, "Superheavy-Higgs-scalar effects in effective gauge theories from $\mathrm{SO}(10)$ grand unification with low-mass right-handed gauge bosons," Physical Review D: Particles, Fields, Gravitation and Cosmology, vol. 40, no. 9, pp. 3074-3085, 1989.

[107] M. K. Parida and B. Sahoo, "Planck-scale induced left-right gauge theory at LHC and experimental tests," Nuclear Physics $B$, vol. 906, pp. 77-104, 2016.

[108] B. Sahoo and M. K. Parida, "Low mass right-handed gauge bosons from minimal grand unified theories," Nuclear and Particle Physics Proceedings, vol. 273-275, pp. 2642-2644, 2016.

[109] Superkamiokande Collaboration, K. Abe, Y. Haga, Y. Hayato, M. Ikeda, K. Iyogi et al., "Search for Proton Decay via $\mathrm{p} \longrightarrow \mathrm{e}+\pi 0$ and $\mathrm{p} \longrightarrow \mu+\pi 0$ in 0.31 Megaton Years Exposoure of Water Cherenkov Detector," Physical Review D, vol. 95, no. 1, Article ID 012004, 2017.

[110] M. K. Parida, B. P. Nayak, R. Satpathy, and R. L. Awasthi, "Standard Coupling Unification in SO(10), Hybrid Seesaw Neutrino Mass, Leptogenesis, Dark Matter, and Proton Lifetime 
Predictions," Journal of High Energy Physics, vol. 1704, no. 75, 2017.

[111] Particle Data Group, J. Beringer et al., "Review of Particle Physics (RPP)," Physical Review D, vol. 86, Article ID 010001, 2012.

[112] M. L. Kynshi and M. K. Parida, "Higgs scalar in the grand desert with observable proton lifetime in $\mathrm{SU}(5)$ and small neutrino masses in SO(10)," Physical Review D: Particles, Fields, Gravitation and Cosmology, vol. 47, no. 11, pp. R4830-R4834, 1993.

[113] K. Olive, K. Agashe, and C. Amsler, "Review of particle physics," Chinese Physics C, vol. 38, no. 9, Article ID 090001, 2014.

[114] K. Olive, "Review of Particle Physics," Chinese Physics C, vol. 40, no. 10, Article ID 100001, 2016.

[115] H. Georgi, H. R. Quinn, and S. Weinberg, "Hierarchy of interactions in unified gauge theories," Physical Review Letters, vol. 33, no. 7, pp. 451-454, 1974.

[116] D. R. Jones, “ Two-loop ," Physical Review D: Particles, Fields, Gravitation and Cosmology, vol. 25, no. 2, pp. 581-582, 1982.

[117] M. K. Parida, "Radiative Seesaw in SO(10) with Dark Matter," Physics Letters B, vol. 704, pp. 206-210, 2011.

[118] R. N. Mohapatra and M. K. Parida, "Type-II seesaw dominance in nonsupersymmetric and split-supersymmetry $\mathrm{SO}(10)$ models and proton lifetime," Physical Review D: Particles, Fields, Gravitation and Cosmology, vol. 84, no. 9, Article ID 095021, 2011.

[119] J. Schechter and J. W. F. Valle, "Neutrinoless double- $\beta$ decay in $\operatorname{su}(2) \mathrm{xu}(1)$ theories," Physical Review D: Particles, Fields, Gravitation and Cosmology, vol. 25, no. 11, article 2951, 1982.

[120] B. P. Nayak and M. K. Parida, "New mechanism for Type-II seesaw dominance in $\mathrm{SO}(10)$ with low-mass $\mathrm{ZI}, \mathrm{RH}$ neutrinos, and verifiable LFV, LNV and proton decay," The European Physical Journal C, vol. 75, article no. 183, 2015.

[121] R. N. Mohapatra and M. K. Parida, "Threshold effects on the mass-scale predictions in $\mathrm{SO}(10)$ models and solar-neutrino puzzle," Physical Review D: Particles, Fields, Gravitation and Cosmology, vol. 47, no. 1, pp. 264-272, 1993.

[122] D.-G. Lee, R. N. Mohapatra, M. K. Parida, and M. Rani, "Predictions for the proton lifetime in minimal nonsupersymmetric SO(10) models: an update," Physical Review D: Particles, Fields, Gravitation and Cosmology, vol. 51, no. 1, pp. 229-235, 1995.

[123] M. Shiozawa, talk presented at TAUP, Asilomar, CA, U.S.A., 8-13 September, 2013.

[124] J. C. Pati, "Advantages of unity with SU(4)-color: Reflections through neutrino oscillations, baryogenesis and proton decay," International Journal of Modern Physics A, vol. 32, no. 09, p. 1741013, 2017.

[125] Superkamiokande Collaboration, H. Nishino et al., "Search for Nucleon Decay into Charged Anti-lepton plus Meson in SuperKamiokande I and II," Physical Review D, vol. 85, Article ID 112001, 2012.

[126] J. L. Raaf, "Recent Nucleon Decay Results from SuperKamiokande," Nuclear Physics B-Proceedings Supplements, vol. 229-232, p. 559, 2012.

[127] K. S. Babu, J. C. Pati, and Z. Tavartkiladze, "Constraining proton lifetime in $\mathrm{SO}(10)$ with stabilized doublet-triplet splitting," Journal of High Energy Physics, vol. 2010, article 84, 2010.

[128] B. Bajc, I. Dorsner, and M. Nemevsek, "Minimal SO(10) Splits Supersymmetry," Journal of High Energy Physics, vol. 811, 2008.

[129] A. Buras, J. Ellis, M. Gaillard, and D. Nanopoulos, "Aspects of the grand unification of strong, weak and electromagnetic interactions," Nuclear Physics B, vol. 135, no. 1, pp. 66-92, 1978.
[130] Y. Aoki, C. Dawson, J. Noaki, and A. Soni, "Proton decay matrix elements with domain-wall fermions," Physical Review D: Particles, Fields, Gravitation and Cosmology, vol. 75, no. 1, 2007.

[131] Y. Aoki, E. Shintani, and A. Soni, "Proton Decay Matrix Elements on the Lattice," Physical Review D, vol. 89, no. 1, Article ID 014505, 2014.

[132] C. Muñoz, "Enhancement factors for supersymmetric proton decay in SU(5) and SO(10) with superfield techniques," Physics Letters B, vol. 177, no. 1, pp. 55-59, 1986.

[133] G. Bertone and D. Hooper, "A History of Dark Matter," Reviews of Modern Physics, 2016.

[134] R. Catena and P. Ullio, "A novel determination of the local dark matter density," Journal of Cosmology and Astroparticle Physics, vol. 1008, no. 004, 2010.

[135] B. W. Lee and S. Weinberg, "Cosmological lower bound on heavy-neutrino masses," Physical Review Letters, vol. 39, no. 4, pp. 165-168, 1977.

[136] K. Griest and M. Kamionkowski, "Unitarity limits on the mass and radius of dark-matter particles," Physical Review Letters, vol. 64, no. 6, pp. 615-618, 1990.

[137] G. Arcadi, M. Lindner, Y. Mambrini, M. Pierre, and F. S. Queiroz, "GUT models at current and future hadron colliders and implications to dark matter searches," Physics Letters B, vol. 771, pp. 508-514, 2017.

[138] Y. Mambrini, S. Profumo, and F. S. Queiroz, "Dark matter and global symmetries," Physics Letters. B. Particle Physics, Nuclear Physics and Cosmology, vol. 760, pp. 807-815, 2016.

[139] P. V. Dong, D. T. Huong, F. S. Queiroz, J. W. Valle, and C. A. Vaquera-Araujo, "The dark side of flipped trinification," Journal of High Energy Physics, vol. 4, p. 143, 2018.

[140] J. M. Cline, K. Kainulainen, P. Scott, and C. Weniger, "Update on scalar singlet dark matter," Physical Review D, vol. 88, Article ID 055025, 2013.

[141] J. M. Cline, K. Kainulainen, P. Scott, and C. Weniger, "Erratum: Update on scalar singlet dark matter," Physical Review D: Particles, Fields, Gravitation and Cosmology, vol. 92, no. 3, 2015.

[142] Z. Ahmed et al., "Results from a Low-Energy Analysis of the CDMS II Germanium Data," Physical Review Letters, vol. 106, Article ID 131302, 2011.

[143] R. Agnese et al., "Improved WIMP-search reach of the CDMS II germanium data," Physical Review D, vol. 92, no. 7, Article ID 072003, 2015.

[144] R. Bernabei, P. Belli, F. Cappella et al., "First results from DAMA/LIBRA and the combined results with DAMA/NaI," The European Physical Journal C, vol. 56, no. 3, pp. 333-355, 2008.

[145] R. Bernabei et al., "New results from DAMA/LIBRA," European Physical Journal C, vol. 67, p. 39, 2010.

[146] M. W. Goodman and E. Witten, "Detectability of certain dark-matter candidates," Physical Review D: Particles, Fields, Gravitation and Cosmology, vol. 31, no. 12, pp. 3059-3063, 1985.

[147] L. Baudis, “Dark matter detection,” Journal of Physics G: Nuclear and Particle Physics, vol. 43, no. 4, p. 044001, 2016.

[148] M. Schumann, L. Baudis, L. Bütikofer, A. Kish, and M. Selvi, "Dark matter sensitivity of multi-ton liquid xenon detectors," Journal of Cosmology and Astroparticle Physics, vol. 1510, no. 10, 2015.

[149] I. Garg, S. Goswami, K. Vishnudath, and N. Khan, "Electroweak vacuum stability in presence of singlet scalar dark matter in 
TeV scale seesaw models," Physical Review D: Particles, Fields, Gravitation and Cosmology, vol. 96, no. 5, 2017.

[150] M. Aguilar et al., "First Result from the Alpha Magnetic Spectrometer on the International Space Station: Precision Measurement of the Positron Fraction in Primary Cosmic Rays of $0.5350 \mathrm{GeV}$," Physical Review Letters, vol. 110, Article ID 141102, 2013.

[151] A. Abramowski et al., "Search for Photon-Linelike Signatures from Dark Matter Annihilations with H.E.S.S," Physical Review Letters, vol. 110, Article ID 041301, 2013.

[152] J. Albert et al., "VHE Gamma-Ray Observation of the Crab Nebula and its Pulsar with the MAGIC telescope," The Astrophysical Journal, vol. 674, p. 1037, 2008.

[153] J. Chang et al., "An excess of cosmic ray electrons at energies of 300-800 GeV," Nature, vol. 456, p. 362, 2008.

[154] P. Athron, C. Balazs, A. Fowlie, and Y. Zhang, "Modelindependent analysis of the DAMPE excess," Journal of High Energy Physics, vol. 1802, no. 121, 2018.

[155] E. Bulbul, M. Markevitch, A. Foster, R. K. Smith, M. Loewenstein, and S. W. Randall, "Detection of an unidentified emission line in the stacked X-ray spectrum of galaxy clusters," The Astrophysical Journal, vol. 789, article 13, 2014.

[156] A. Boyarsky, O. Ruchayskiy, D. Iakubovskyi, and J. Franse, "Unidentified line in X-ray spectra of the andromeda galaxy and perseus galaxy cluster," Physical Review Letters, vol. 113, no. 25, Article ID 251301, 2014.

[157] T. Bringmann, X. Huang, A. Ibarra, S. Vogl, and C. Weniger, "Fermi LAT search for internal bremsstrahlung signatures from dark matter annihilation," Journal of Cosmology and Astroparticle Physics, vol. 1207, p. 054, 2012.

[158] C. Weniger, "A tentative gamma-ray line from Dark Matter annihilation at the Fermi Large Area Telescope," Journal of Cosmology and Astroparticle Physics, vol. 1208, no. 007, 2012.

[159] T. Daylan, D. P. Finkbeiner, D. Hooper et al., "The characterization of the gamma-ray signal from the central Milky Way: A case for annihilating dark matter," Physics of the Dark Universe, vol. 12, pp. 1-23, 2016.

[160] T. Jeltema and S. Profumo, "Discovery of a $3.5 \mathrm{keV}$ line in the Galactic Centre and a critical look at the origin of the line across astronomical targets," Monthly Notices of the Royal Astronomical Society, vol. 450, no. 2, pp. 2143-2152, 2015.

[161] J. Petrović, P. D. Serpico, and G. Zaharijaš, "Galactic Center gamma-ray "excess" from an active past of the Galactic Centre?" Journal of Cosmology and Astroparticle Physics, vol. 1410, no. 10, p. 052, 2014.

[162] M. Gonderinger, Y. Li, H. Patel, and M. J. Ramsey-Musolf, "Vacuum stability, perturbativity, and scalar singlet dark matter," Journal of High Energy Physics, vol. 1001, no. 53, 2010.

[163] C. Chen and Y. Tang, "Vacuum stability, neutrinos, and dark matter," Journal of High Energy Physics, vol. 1204, no. 19, 2012.

[164] N. Khan and S. Rakshit, "Study of electroweak vacuum metastability with a singlet scalar dark matter," Physical Review D: Particles, Fields, Gravitation and Cosmology, vol. 90, no. 11, 2014.

[165] P. Athron et al., "Status of the scalar singlet dark matter model," The European Physical Journal C, vol. 77, no. 8, p. 568, 2017.

[166] M. Cirelli, A. Strumia, and M. Tamburini, "Cosmology and astrophysics of minimal dark matter," Nuclear Physics B, vol. 787, no. 1-2, pp. 152-175, 2007.

[167] A. Arhrib, R. Benbrik, M. Chabab et al., "Higgs potential in the type II seesaw model," Physical Review D: Particles, Fields, Gravitation and Cosmology, vol. 84, no. 9, 2011.
[168] N. Haba, H. Ishida, N. Okada, and Y. Yamaguchi, "Vacuum stability and naturalness in type-II seesaw," The European Physical Journal C, vol. 76, no. 6, 2016.

[169] D. Chang, R. N. Mohapatra, and M. K. Parida, "New mechanism for baryon generation in $\mathrm{SO}(10)$ models with low mass WRboson," Physics Letters B, vol. 142, no. 1-2, pp. 55-58, 1984.

[170] R. Satpathy, M. Chakraborty, B. Sahoo, M. K. Parida, Work in progress. 

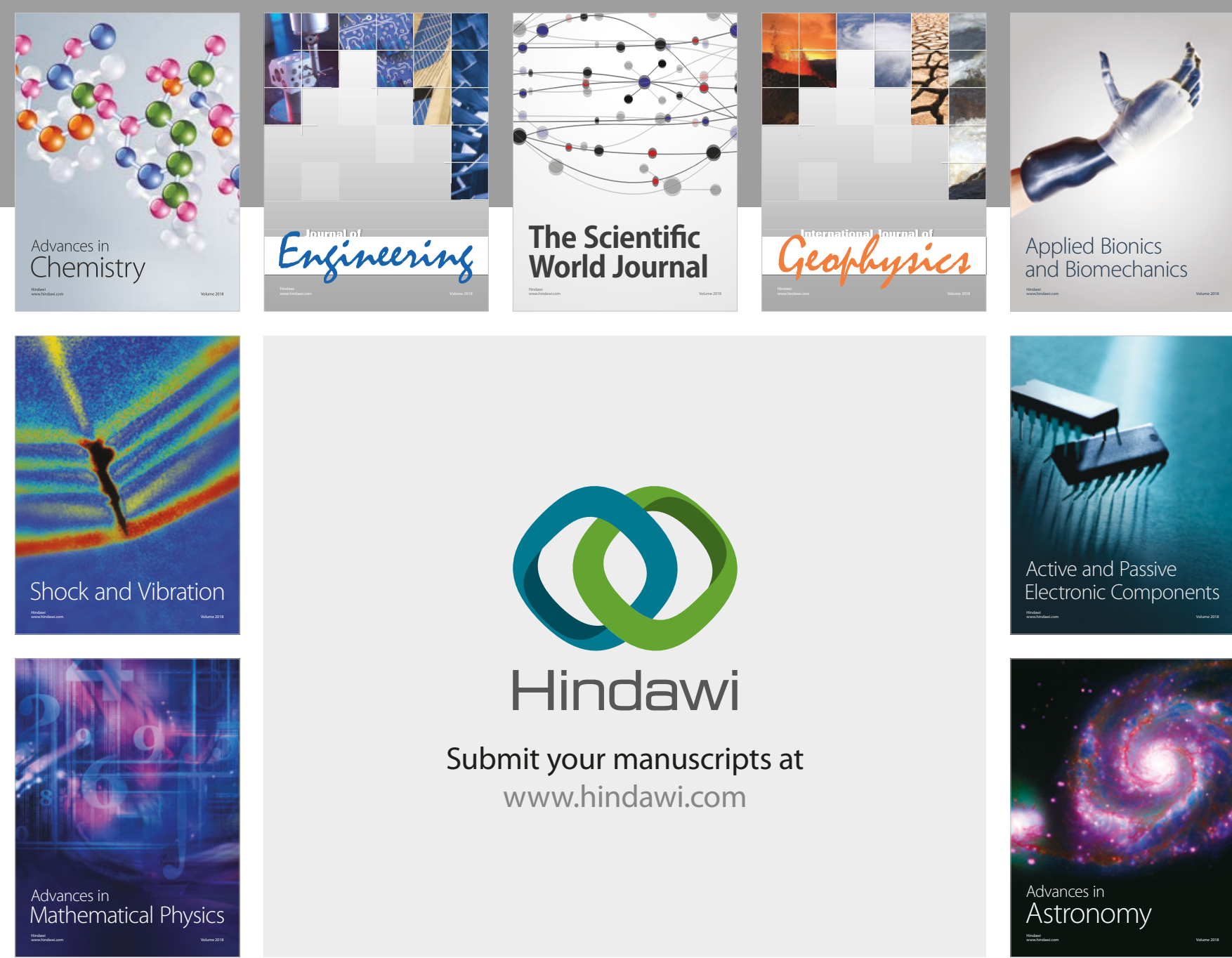

Submit your manuscripts at

www.hindawi.com

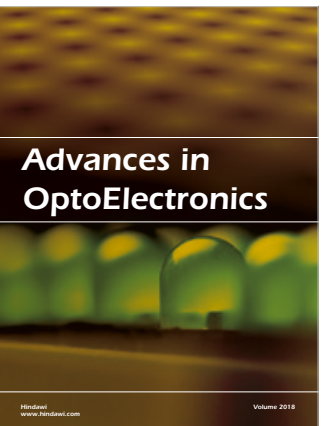

\section{Rotcting Machinery}
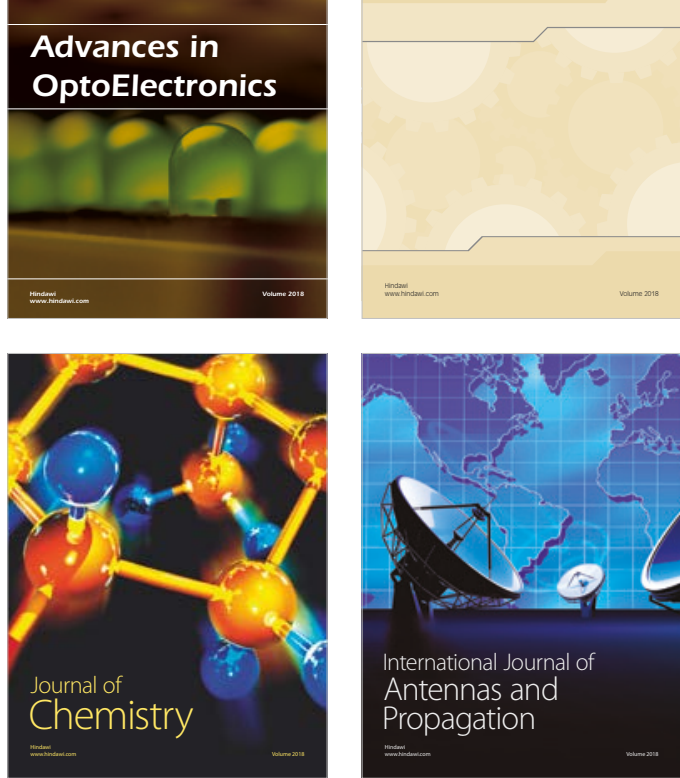

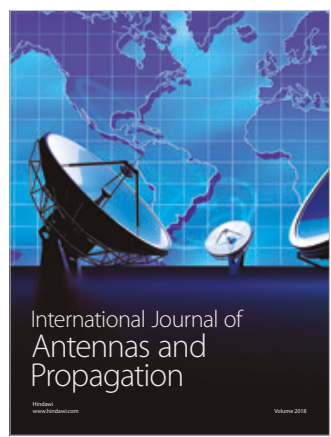

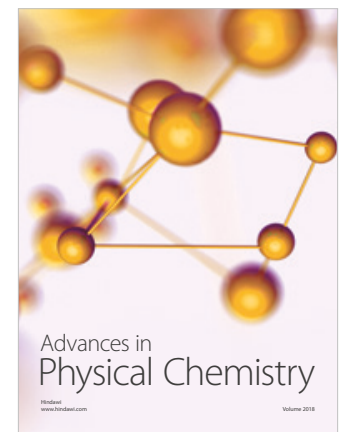

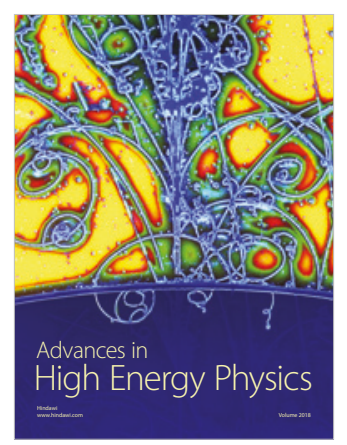

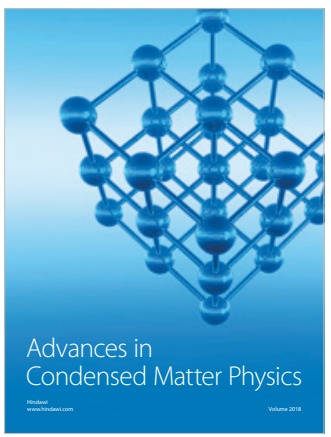

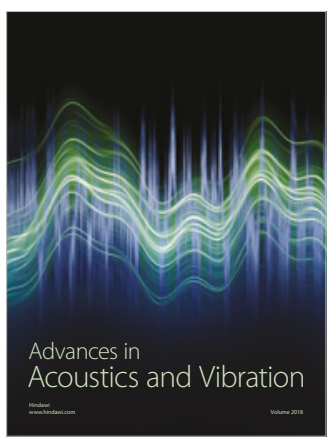

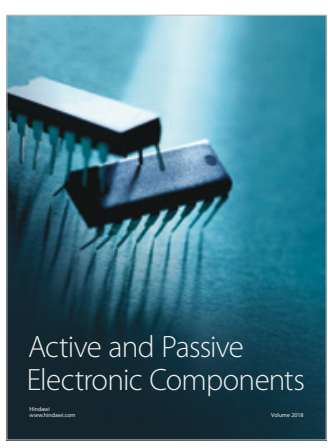
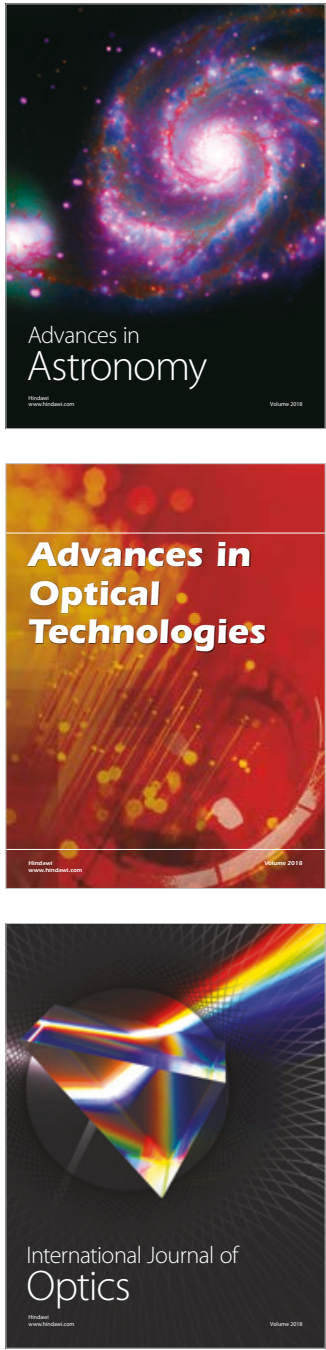